\title{
Holonomy classification of Lorentz-Kähler manifolds
}

\author{
Anton S. Galaev
}

\begin{abstract}
The classification problem for holonomy of pseudo-Riemannian manifolds is actual and open. In the present paper, holonomy algebras of Lorentz-Kähler manifolds are classified. A simple construction of a metric for each holonomy algebra is given. Complex Walker coordinates are introduced and described using the potential. Complex pp-waves are characterized in terms of the curvature, holonomy and the potential. Classification of Lorentz-Kähler symmetric spaces is reviewed.
\end{abstract}

Keywords: Lorentz-Kähler manifold; holonomy group; complex Walker coordinates; complex pp-wave; symmetric space; space of oriented lines

2010 Mathematical Subject Codes: 53C29, 53C25, 53C $35,53 \mathrm{C} 50,53 \mathrm{C} 55$

\section{Contents}

1. Introduction

2. Preliminaries

3. The Classification Theorem

4. Weakly irreducible subalgebras in $\mathfrak{u}(1, n+1)$

5. Algebraic curvature tensors and Berger algebras

6. Lorentz-Kähler metrics

7. Example: the space of oriented lines in $\mathbb{R}^{3}$

8. Complex pp-waves 19

9. Lorentz-Kähler symmetric spaces 20

References

\section{Introduction}

The holonomy group of a pseudo-Riemannian manifold $(M, g)$ is an important invariant that gives rich information about the geometry of $(M, g)$. This motivates the classification problem for holonomy groups of pseudo-Riemannian manifolds. The problem is solved only for connected holonomy groups of Riemannian and Lorentzian manifolds. The case of Riemannian manifolds is classical and it has many applications in differential geometry and theoretical physics, see e.g. [9, 14, 34. The case of Lorentzian manifolds took the attention of geometers and theoretical physicists during the last two decades, see the reviews $\mathbf{3}, \mathbf{2 5}, \mathbf{2 6}$ ] and the references therein. In the other signatures, only partial results are known $[\mathbf{1 2}, \mathbf{1 3}, \mathbf{7}, \mathbf{2 3}, \mathbf{2 4}, \mathbf{2 8}, \mathbf{2 9}, \mathbf{3 5}$. The main difference between holonomy groups of Riemannian and proper pseudo-Riemannian manifolds is that for Riemannian manifolds all considerations may be reduced to the case of irreducible holonomy groups, while in the case of proper pseudo-Riemannian manifolds one should consider also holonomy groups preserving degenerate subspaces. Berger 10 classified connected irreducible holonomy groups of pseudo-Riemannian manifolds of arbitrary signature. Note that there is a

University of Hradec Králové, Faculty of Science, Rokitanského 62, 50003 Hradec Králové, Czech Republic E-mail: anton.galaev(at)uhk.cz. 
more general classification of connected irreducible holonomy groups of torsion-free affine connections $[40$.

The general case of the signature $(2, N)$, i.e., the next signature after the Lorentzian one, is quite complicated, this show the work [35. In the present paper we give a complete classification of the connected holonomy groups (equivalently, of holonomy algebras) for pseudo-Kähler manifolds of complex index one; such manifolds are called Lorentz-Kähler and they have holonomy algebras contained in $\mathfrak{u}(1, n+1) \subset \mathfrak{s o}(2,2 n+2)$ (here $n+2$ is the complex dimension of the manifold).

We do the same three steps that were previously done for the holonomy of Lorentzian manifolds [8, 37, 27. The Wu theorem allows to assume that the holonomy algebra is weakly irreducible (this means that it does not preserve any proper non-degenerate subspace of the tangent space). Since the case of irreducible holonomy algebras is solved by Berger, we assume that the holonomy algebra is not irreducible, and then (with one exception in dimension four) it preserves a complex isotropic line. In Section 4 we classify weakly irreducible subalgebras in $\mathfrak{u}(1, n+1)$. For that we consider the following geometrical approach: we consider the induced action of the corresponding connected Lie subgroup $G \subset \mathrm{U}(1, n+1)$ on the boundary of the complex hyperbolic space, which may be identified with the sphere. This action induces an action of $G$ on the Heisenberg space and on the Hermitian space $\mathbb{C}^{n}$. Then $G$ preserves a real affine subspace $L \subset \mathbb{C}^{n}$, where it acts transitively as the group of similarity transformations. This allows to find all groups $G$. Using this, in Section [5 we classify weakly irreducible Berger subalgebras in $\mathfrak{u}(1, n+1)$, i.e. weakly irreducible subalgebras in $\mathfrak{u}(1, n+1)$ having the same algebraic properties as the holonomy algebras. Berger algebras play the role of the candidates to the holonomy algebras.

Then appears the most important problem to check, which of these candidates are really holonomy algebras. Note that in the case of Riemannian manifolds the same problem was open for more than thirty years, and finally it was solved by Bryant [15. In Section 6 we show that some of the obtained by us Berger algebras cannot appear as the holonomy algebras (note that all previously known Berger algebras were always holonomy algebras), and we construct metrics realizing all the other Berger algebras as holonomy algebras. We obtain the metrics by writing down explicitly their potentials, which are relatively simple.

In Section 6 we also introduce the complex version of the Walker coordinates, which are frequently used in Lorentzian geometry and Relativity [16.

Then we consider several examples. In Section 7 we find the holonomy algebra of the space of oriented lines in $\mathbb{R}^{3}$, which admits the structure of the Lorentz-Kähler manifold of real dimension four; this space is used in geometric optics, see the survey [31] and the references therein. There are generalizations of this space giving farther examples of Lorentz-Kähler metrics [31, 32, 33.

The pp-wave metrics are very useful in theoretical physics. In Section 8 we consider their generalization to the complex case. We give equivalent conditions in terms of the curvature, holonomy and the potential for a complex Walker metric to be a complex pp-wave. Complex pp-waves were studied in [39, where appear also examples of Lorentz-Kähler metrics and their holonomy. Complex pp-waves are used also in the physical literature, e.g. [20, 42].

Simply connected pseudo-Riemannian symmetric spaces of index 2 were classified in [36], and the case of Lorentz-Kähler manifolds was considered separately. In Section 9 we reformulate the last result in terms of the curvature and holonomy.

Let us mention the works and situations, where Lorentz-Kähler manifolds and their holonomy algebras appear. There are various examples of invariant Lorentz-Kähler metrics on Lie groups and homogeneous spaces, e.g. $[\mathbf{6}, \mathbf{1 7}, \mathbf{1 8}, \mathbf{4 1}, \mathbf{4 4}$. In some cases the holonomy groups are computed. Lorentz-Kähler metrics and their holonomy are related to conformal geometry, conformal holonomy, and CR-structures through the ambient metric construction, see e.g. [5, 19, 22., 38, in particular, to the conformal analog of Calabi-Yau manifolds 4 .

Completing the introduction, we note that the problem solved in the present paper was considered in the unpublished $\mathrm{PhD}$ thesis $[\mathbf{2 8}$, of the author. The classification result from $2 \mathbf{2 8}$ with a sketch of the proof was given in the survey 24, see remark in Section 3. The exposition of the present paper is much simpler than that in [28], in particular, the most important is the new construction of metrics given in the present paper. 
Acknowledgements. The author is grateful to Helga Baum and Dmitri V. Alekseevsky for useful discussions and suggestions. This work was supported by the Specific Research Project of Faculty of Science, University of Hradec Králové [No. 2101, 2017] and by the grant no. 18-00496S of the Czech Science Foundation.

\section{Preliminaries}

The theory of the holonomy groups of pseudo-Riemannian manifolds can be found e.g. in [9, 34, 24. Let $(M, g)$ be a pseudo-Riemannian manifold of signature $(r, s)$. The holonomy group $G_{x}$ of $(M, g)$ at a point $x \in M$ is the Lie group that consists of the pseudo-orthogonal transformations of the tangent space given by the parallel transports along all piecewise smooth loops at the point $x$. This group may be identified with a Lie subgroup of the pseudo-orthogonal group $\mathrm{O}(r, s)$. The corresponding subalgebra of the pseudo-orthogonal Lie algebra $\mathfrak{s o}(r, s)$ is called the holonomy algebra and it determines the holonomy group if the manifold is simply connected. The Ambrose-Singer Theorem states that the holonomy algebra is spanned by the endomorphisms $\tau_{\gamma}^{-1} \circ R_{y}\left(\tau_{\gamma} X, \tau_{\gamma} Y\right) \circ \tau_{\gamma}$ of $T_{x} M$, where $\gamma$ is a piecewise smooth curve starting at the point $x$ with an end-point $y \in M$, and $X, Y \in T_{x} M$. This implies that the holonomy algebra satisfies a strong algebraic condition being spanned by images of the algebraic curvature tensors; algebras satisfying this property are called Berger algebras (see below), and they are candidates to the holonomy algebras.

The fundamental principle for holonomy groups states that there exists a one-to-one correspondents between parallel tensor fields $T$ on $(M, g)$ and tensors $T_{0}$ of the same type at $x$ preserved by the tensor extension of the representation of the holonomy group.

A subgroup (resp. subalgebra) of the pseudo-orthogonal Lie group (resp. algebra) is called weakly irreducible if it does not preserve any non-degenerate proper subspace of the pseudoEuclidean space). The $\mathrm{Wu}$ decomposition theorem implies that if the holonomy algebra of a pseudo-Riemannian manifold is not weakly irreducible, then the manifold is locally decomposable, and the holonomy algebra is the direct sum of weakly irreducible holonomy algebras. This allows us to assume that the holonomy algebra is weakly irreducible. Irreducible holonomy algebras of pseudo-Riemannian manifolds were classified by Berger [10, so we are left with weakly irreducible not irreducible holonomy algebras.

A pseudo-Kähler manifold is a pseudo-Riemannian manifold $(M, g)$ with a $g$-orthogonal complex structure $J$ that is parallel. By the fundamental principle, the equivalent condition is that the holonomy group of $(M, g)$ is contained in the pseudo-unitary group $\mathrm{U}\left(\frac{r}{2}, \frac{s}{2}\right)$ (the numbers $r$ and $s$ must be even). Together with $J, M$ becomes a complex manifold; the tensor fields $g$ and $J$ define a pseudo-Kähler metric $h$ on $M$ of complex signature $\left(\frac{r}{2}, \frac{s}{2}\right)$. We are interested in holonomy groups of pseudo-Kähler manifold of real signature $(2,2 N)$, i.e. of complex signature $(1, N)$. Such manifolds are called Lorentz-Kähler manifolds. Let $N=n+1, n \geq 0$. The tangent space of a Lorentz-Kähler manifold may be identified with the pseudo-Hermitian space $\mathbb{C}^{1, n+1}$ with a pseudo-Hermitian metric $h$. From the Berger classification it follows that the only irreducible holonomy algebras of Lorentz-Kähler manifolds are $\mathfrak{u}(1, n+1)$ and $\mathfrak{s u}(1, n+1)$. In fact, these Lie algebras exhaust irreducible subalgebras in $\mathfrak{u}(1, n+1)$ [21].

Fix a Witt basis $p, e_{1}, \ldots, e_{n}, q$ of $\mathbb{C}^{1, n+1}$, with respect to such a basis the non-zero values of the metric $h$ are the following: $h(p, q)=h\left(e_{j}, e_{j}\right)=1$. In particular, the vectors $p$ and $q$ are isotropic, and the vectors $e_{1}, \ldots, e_{n}$ form a basis of the Hermitian space, which we denote by $\mathbb{C}^{n}$. We will be interested in the subalgebra $\mathfrak{u}(1, n+1)_{\mathbb{C} p}$ of $\mathfrak{u}(1, n+1)$ preserving the complex isotropic line $\mathbb{C} p$. In the matrix form we have

$$
\mathfrak{u}(1, n+1)_{\mathbb{C} p}=\left\{\left(\begin{array}{ccc}
a & -\bar{Z}^{t} & i c \\
0 & A & Z \\
0 & 0 & -\bar{a}
\end{array}\right) \mid \begin{array}{c}
a \in \mathbb{C}, A \in \mathfrak{u}(n), \\
Z \in \mathbb{C}^{n} \\
c \in \mathbb{R}
\end{array}\right\} .
$$

We will denote the above matrix by the 4 -tuple $(a, A, Z, c)$. For the Lie brackets we have

$[(a, A, 0,0),(b, B, Z, c)]=\left(0,[A, B]_{\mathfrak{u}(n)}, \bar{a} Z+A Z, 2 i c \operatorname{Re} a\right), \quad[(0,0, Z, 0),(0,0, V, c)]=(0,0,0,2 i \operatorname{Im} h(Z, V))$. 
We obtain the decomposition

$$
\mathfrak{u}(1, n+1)_{\mathbb{C} p}=(\mathbb{C} \oplus \mathfrak{u}(n)) \ltimes\left(\mathbb{C}^{n} \ltimes i \mathbb{R}\right) .
$$

Note that the subalgebra $\mathbb{C}^{n} \ltimes i \mathbb{R} \subset \mathfrak{u}(1, n+1)_{\mathbb{C} p}$ is isomorphic to the Heisenberg Lie algebra, and the subalgebra $\mathfrak{s u}(1, n+1)_{\mathbb{C} p} \subset \mathfrak{u}(1, n+1)_{\mathbb{C} p}$ is isomorphic to the Lie algebra of the similarity transformations of the Heisenberg space. We will denote the complex structure on $\mathbb{C}^{1, n+1}$ by $J$.

We will consider real vector subspaces of $\mathbb{C}^{n}$ of the form

$$
L=\mathbb{C}^{m} \oplus L_{0},
$$

where $0 \leq m \leq n$, we fix an $h$-orthogonal decomposition $\mathbb{C}^{n}=\mathbb{C}^{m} \oplus \mathbb{C}^{n-m}$, and $L_{0} \subset \mathbb{C}^{n-m}$ is a real form, i.e. $i L_{0} \cap L_{0}=0, L_{0} \oplus i L_{0}=\mathbb{C}^{n-m}$. An example of $L_{0}$ is

$$
\mathbb{R}^{n-m}=\operatorname{span}_{\mathbb{R}}\left\{e_{m+1}, \ldots, e_{n}\right\} .
$$

Note that in general $L_{0}$ is different from this one, since $L_{0}$ may not contain an $h$-orthonormal basis of $\mathbb{C}^{n-m}$.

For the case $n=0$ let us consider the subalgebra $\mathfrak{g}_{0} \subset \mathfrak{s u}(1,1)$ defined in the following way. Consider the Witt basis $p_{1}, p_{2}, q_{1}, q_{2}$ in $\mathbb{R}^{2,2}$ and define the pseudo-Hermitian structure $J$ such that $J p_{1}=q_{2}, J p_{2}=-q_{1}$. Let

$$
\mathfrak{g}_{0}=\left\{\left(\begin{array}{cc}
A & 0 \\
0 & -A^{t}
\end{array}\right) \mid A \in \mathfrak{s l}(2, \mathbb{R})\right\} \subset \mathfrak{s o}(2,2) .
$$

The Lie algebra $\mathfrak{g}_{0}$ commutes with $J$, hence it is contained in $\mathfrak{u}(1,1)$. Moreover, $\mathfrak{g}_{0} \subset \mathfrak{s u}(1,1)$. The Lie algebra $\mathfrak{g}_{0}$ preserves the vector subspace $\operatorname{span}_{\mathbb{R}}\left\{p_{1}, p_{2}\right\}$, which is not $J$-invariant.

\section{The Classification Theorem}

TheOREM 3.1. 1) A subalgebra $\mathfrak{g} \subset \mathfrak{u}(1,1)$ is the weakly irreducible not irreducible holonomy algebra of a Lorentz-Kähler manifold of complex dimension 2 if and only if $\mathfrak{g}$ is conjugated to $\mathfrak{g}_{0}$, or $\mathfrak{g}$ is conjugated to one of the following subalgebras of $\mathfrak{u}(1,1)_{\mathbb{C} p}$ :

- $\mathfrak{g}_{1}=\mathfrak{u}(1,1)_{\mathbb{C} p}$

- $\mathfrak{g}_{2}=\left\{\left(\begin{array}{cc}a & 0 \\ 0 & -\bar{a}\end{array}\right) \mid a \in \mathbb{C}\right\}$;

- $\mathfrak{g}_{3}^{\gamma}=\left\{\left(\begin{array}{cc}r \gamma & i c \\ 0 & -r \bar{\gamma}\end{array}\right) \mid r, c \in \mathbb{R}\right\}$, where $\gamma \in \mathbb{C}$ is a fixed number.

2) Let $n \geq 1$. Then a subalgebra $\mathfrak{g} \subset \mathfrak{u}(1, n+1)$ is the weakly irreducible not irreducible holonomy algebra of a Lorentz-Kähler manifold of complex dimension $n+2$ if and only if $\mathfrak{g}$ is conjugated to one of the following subalgebras of $\mathfrak{u}(1, n+1)_{\mathbb{C} p}$ :

- $\mathfrak{g}^{\mathfrak{k}}=\mathfrak{k} \ltimes\left(\mathbb{C}^{n} \ltimes i \mathbb{R}\right)$

$$
=\left\{\left(\begin{array}{ccc}
a & -\bar{Z}^{t} & i c \\
0 & A & Z \\
0 & 0 & -\bar{a}
\end{array}\right) \mid \begin{array}{c}
a+A \in \mathfrak{k}, \\
Z \in \mathbb{C}^{n} \\
c \in \mathbb{R}
\end{array}\right\},
$$

where

$$
\mathfrak{k} \subset \mathbb{C} \oplus \mathfrak{u}(n)
$$

is an arbitrary subalgebra;

$$
\begin{gathered}
\cdot \mathfrak{g}^{\mathfrak{k}, J, L}=\mathfrak{k} \ltimes(L \ltimes i \mathbb{R}) \\
\left.=\left\{\begin{array}{cccc}
a_{2} i & -\bar{Z}^{t} & -\bar{X}^{t} & i c \\
0 & A & 0 & Z \\
0 & 0 & a_{2} i E_{n-m} & X \\
0 & 0 & 0 & a_{2} i
\end{array}\right) \mid \begin{array}{c}
a_{2}\left(i+i \operatorname{id}_{\mathbb{C}^{n-m}}\right)+A \in \mathfrak{k}, \\
Z \in \mathbb{C}^{m}, X \in \mathbb{R}^{n-m}, \\
c \in \mathbb{R}
\end{array}\right\}, \\
\text { where } L=\mathbb{C}^{m} \oplus \mathbb{R}^{n-m}, 0 \leq m<n, \\
\mathfrak{k} \subset \mathbb{R} J \oplus \mathfrak{u}(m)
\end{gathered}
$$

is an arbitrary subalgebra not contained in $\mathfrak{u}(m)$; 
- $\mathfrak{g}^{\mathfrak{k}, L}=\mathfrak{k} \ltimes(L \ltimes i \mathbb{R})$

$=\left\{\left(\begin{array}{cccc}0 & -\bar{Z}^{t} & -\bar{X}^{t} & i c \\ 0 & A & 0 & Z \\ 0 & 0 & 0 & X \\ 0 & 0 & 0 & 0\end{array}\right) \mid \begin{array}{c}A \in \mathfrak{k}, \\ Z \in \mathbb{C}^{m}, X \in L_{0}, \\ c \in \mathbb{R}\end{array}\right\}$,

where $L=\mathbb{C}^{m} \oplus L_{0}, 0 \leq m<n, L_{0} \subset \mathbb{C}^{n-m}$ is a real form,

$$
\mathfrak{k} \subset \mathfrak{u}(m)
$$

is an arbitrary subalgebra;

- $\mathfrak{g}^{\mathfrak{k}_{0}, \psi}=\mathfrak{k}_{0} \oplus\left\{(0, \psi(X), X, 0) \mid X \in \mathbb{C}^{m-r} \oplus L_{0}\right\} \ltimes\left(\mathbb{C}^{r} \ltimes i \mathbb{R}\right)$

$$
=\left\{\left(\begin{array}{cccc}
0 & -\bar{Z}^{t} & -\bar{X}^{t} & i c \\
0 & A+\psi(X) & 0 & Z \\
0 & 0 & 0 & X \\
0 & 0 & 0 & 0
\end{array}\right) \mid \begin{array}{c}
A \in \mathfrak{k}_{0}, \\
Z \in \mathbb{C}^{r}, X \in \mathbb{C}^{m-r} \oplus L_{0}, \\
c \in \mathbb{R}
\end{array}\right\},
$$

where $1 \leq r \leq m \leq n, L=\mathbb{C}^{m} \oplus L_{0}, L_{0} \subset \mathbb{C}^{n-m}$ is a real form, we consider the decomposition

$$
\mathbb{C}^{m}=\mathbb{C}^{r} \oplus \mathbb{C}^{m-r}
$$

$\mathfrak{k}_{0} \subset \mathfrak{u}(r)$ is an arbitrary subalgebra,

$$
\psi: \mathbb{C}^{m-r} \oplus L_{0} \rightarrow \mathfrak{u}(r)
$$

is a non-zero linear map such that the image $\psi\left(\mathbb{C}^{m-r} \oplus L_{0}\right) \subset \mathfrak{u}(r)$ is commutative, it commutes with $\mathfrak{k}_{0}$ and has trivial intersection with $\mathfrak{k}_{0}$.

Recall that, by definition, a special pseudo-Kähler manifold (or Calabi-Yau manifold of indefinite signature) is just a Ricci-flat pseudo-Kähler manifold. Special pseudo-Kähler manifolds are precisely pseudo-Kähler manifolds with holonomy algebras contained in the special unitary Lie algebra. We imminently get the following corollary.

COROLlary 3.1. Weakly irreducible not irreducible holonomy algebras of Ricci-flat LorentzKähler manifolds are exhausted by the following algebras:

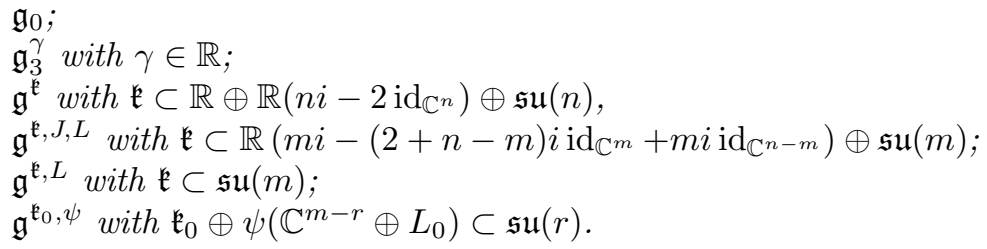

\section{Proof of Theorem 3.1.}

Suppose that $\mathfrak{g} \subset \mathfrak{u}(1, n+1)$ is the weakly irreducible not irreducible holonomy algebra of a Lorentz-Kähler manifold. Then $\mathfrak{g}$ preserves a proper real subspace $W \subset \mathbb{R}^{2,2 n+2}=\mathbb{C}^{1, n+1}$. Consequently $\mathfrak{g}$ preserves the orthogonal complement $W^{\perp} \subset \mathbb{R}^{2,2 n+2}$ and the intersection $W \cap W^{\perp}$, which is isotropic and must be of real dimension 1 or 2 . Let $W_{1}=\left(W \cap W^{\perp}\right)^{\perp}$.

Suppose that $n=0$. If $W_{1} \cap J W_{1} \neq 0$, then $\mathfrak{g}$ is conjugated to a subalgebra of $\mathfrak{u}(1,1)_{\mathbb{C} p}$. It is not hard to write down all subalgebras of $\mathfrak{u}(1,1)_{\mathbb{C} p}$ and decide which of them are weakly irreducible Berger subalgebras in $\mathfrak{u}(1,1)_{\mathbb{C} p}$, i.e. weakly irreducible subalgebras in $\mathfrak{u}(1,1) \mathbb{C}_{p}$ having the same algebraic properties as the holonomy algebras. In Section 6 we construct metrics with each of the obtained algebras being the holonomy algebra. If $W_{1} \cap J W_{1}=0$, then it is not hard to see that $\mathfrak{g}$ is contained in $\mathfrak{g}_{0}$. Holonomy algebras of pseudo-Riemannian manifolds of signature $(2,2)$ are well studied [7, 29, by this reason we do not pay much attention to the case $n=0$.

Suppose that $n>0$. Then $W_{2}=W_{1} \cap J W_{1}$ is non-trivial, $\mathfrak{g}$ - and $J$-invariant, and degenerate. Thus, $\mathfrak{g}$ preserves the complex isotropic line $W_{2} \cap W_{2}^{\perp}$. Hence $\mathfrak{g}$ is conjugated to a subalgebra of $\mathfrak{u}(1, n+1) \mathbb{C} p$.

The next three sections will be dedicated to the rest of the proof of Theorem 3.1 In Section 4 we will describe weakly irreducible subalgebras in $\mathfrak{u}(1, n+1)_{\mathbb{C} p}$. Using this, in Section 5 we classify weakly irreducible Berger subalgebras in $\mathfrak{u}(1, n+1) \mathbb{C} p$. In Section 6 we show that some of 
the obtained Berger algebras cannot appear as the holonomy algebras, and we construct metrics realizing all the other Berger algebras as holonomy algebras. This will prove Theorem 3.1 .

Remark. The first attempt to classify holonomy algebras of Lorentz-Kähler manifolds was done in the unpublished $\mathrm{PhD}$ thesis [28] of the author. The exposition of [28] is very complicated compared to the exposition of the present paper, e.g., the metrics constructed in [28] were given in real coordinates and in a very involved way. In [28, it was wrongly assumed that any real form $L \subset \mathbb{C}^{n}$ contains an orthonormal basis for the Hermitian form on $\mathbb{C}^{n}$, i.e., the endomorphism $\theta$ defined below was assumed to be zero. The statement of Theorem 6.1 given below was not discovered in [28. The classification result from [28] with a sketch of the proof was given in the survey [24] (Theorem 4.2). Let us compare [24, Th. 4.2] with the above Theorem 3.1] in the case $n \geq 1$. The results of the present paper imply that each of the first five algebras $\mathfrak{h}$ from 24 , Th. 4.2, part 2] must satisfy the condition: if $\mathfrak{h} \not \subset(\mathbb{R} J \oplus \mathfrak{u}(n)) \ltimes\left(\mathbb{C}^{n} \ltimes i \mathbb{R}\right)$, then the number $m$ associated to $\mathfrak{h}$ is equal to $n$ (this is the consequence of Theorem 6.1 given below). Under that condition these algebras exhaust the first two algebras from part 2 of Theorem 3.1 The third algebra from part 2 of Theorem 3.1 that satisfies the condition $L_{0} \neq \mathbb{R}^{n-m}$ (i.e., with $\theta \neq 0$ ) is missing in [24, Th. 4.2]. If $L_{0}=\mathbb{R}^{n-m}$ (i.e., $\theta=0$ ) then this is the third algebra from [24, Th. 4.2, part 2] with $\varphi=\phi=0$. Likewise, the last two algebras from [24, Th. 4.2] correspond to the last algebra from Theorem 3.1 with $L_{0}=\mathbb{R}^{n-m}$.

\section{Weakly irreducible subalgebras in $\mathfrak{u}(1, n+1)$}

Let $\mathrm{U}(1, n+1)_{\mathbb{C} p}$ be the Lie subgroup of $\mathrm{U}(1, n+1)$ preserving the line $\mathbb{C} p$. This group is generated by the elements of the form:

$$
\left(\begin{array}{ccc}
e^{a} & 0 & 0 \\
0 & A & 0 \\
0 & 0 & e^{-\bar{a}}
\end{array}\right), \quad\left(\begin{array}{ccc}
1 & -\bar{Z}^{t} & -\frac{1}{2} \bar{Z}^{t} Z+i c \\
0 & E_{n} & Z \\
0 & 0 & 1
\end{array}\right),
$$

where $a \in \mathbb{C}, A \in \mathrm{U}(n), Z \in \mathbb{C}^{n}$, and $c \in \mathbb{R}$.

Consider the group

$$
\operatorname{Sim} \mathbb{C}^{n}=\left(\mathbb{R}^{*} \cdot U(n)\right)<\mathbb{C}^{n}
$$

of similarity transformations of the Hermitian space $\mathbb{C}^{n}$ and the group homomorphism

$$
\Gamma: \mathrm{U}(1, n+1)_{\mathbb{C} p} \rightarrow \operatorname{Sim} \mathbb{C}^{n}
$$

that sends the above elements of $\mathrm{U}(1, n+1)_{\mathbb{C} p}$ to $e^{\bar{a}} \cdot A \in \mathbb{R}^{*} \cdot U(n)$, and $Z \in \mathbb{C}^{n}$, respectively.

The homomorphism $\Gamma$ can be constructed in the following geometrical way. The group $\mathrm{U}(1, n+$ 1) acts in the natural way on the boundary $\partial \mathbf{H}^{n+1}$ of the complex hyperbolic space, which consists of complex isotropic lines in $\mathbb{C}^{1, n+1}\left[\mathbf{3 0}\right.$. The boundary may be identified with the sphere $S^{2 n+1}$. Since the group $\mathrm{U}(1, n+1)_{\mathbb{C} p}$ preserves the point $\{\mathbb{C} p\} \in \partial \mathbf{H}^{n+1}$, it acts on the sphere with one removed point; this space may be identified with the Heisenberg space

$$
\mathbb{C}^{n} \oplus \mathbb{R}
$$

and the action there is given by the Heisenberg similarity transformations. Then we may consider the induced action on $\mathbb{C}^{n}$, which will give us the homomorphism $\Gamma$.

The differential

$$
\Gamma^{\prime}: \mathfrak{u}(1, n+1)_{\mathbb{C} p} \rightarrow \mathfrak{s i m} \mathbb{C}^{n}=(\mathbb{R} \oplus \mathfrak{u}(n)) \ltimes \mathbb{C}^{n}
$$

is given by

$$
(a, A, Z, c) \mapsto\left(\operatorname{Re} a,-i \operatorname{Im} a \operatorname{id}_{\mathbb{C}^{n}}+A, Z\right) .
$$

The kernel of $\Gamma^{\prime}$ equals to $\mathbb{R} J \oplus i \mathbb{R} \subset \mathfrak{u}(1, n+1) \mathbb{C} p$.

Suppose that $G \subset \mathrm{U}(1, n+1) \mathbb{C} p$ is a weakly irreducible Lie subgroup in the sense that it does not preserve any non-degenerate complex subspace in $\mathbb{C}^{1, n+1}$.

Proposition 4.1. The subgroup $\Gamma(G) \subset \operatorname{Sim} \mathbb{C}^{n}$ does not preserve any proper complex affine subspace of $\mathbb{C}^{n}$. Consequently, if $\Gamma(G) \subset \operatorname{Sim} \mathbb{C}^{n}$ preserves a proper real affine subspace $L \subset \mathbb{C}^{n}$, then the minimal complex affine subspace of $\mathbb{C}^{n}$ containing $L$ is $\mathbb{C}^{n}$. 
Proof. First we prove that $\Gamma(G) \subset \operatorname{Sim} \mathbb{C}^{n}$ does not preserve any proper complex vector subspace of $\mathbb{C}^{n}$. Suppose that $\Gamma(G)$ preserves a proper complex vector subspace $L \subset \mathbb{C}^{n}$. Then,

$$
\Gamma(G) \subset\left(\mathbb{R}^{*} \cdot U(L) \cdot U\left(L^{\perp}\right)\right) \curlywedge L,
$$

where $L^{\perp}$ is the orthogonal complement to $L$ in $\mathbb{C}^{n}$. We see that the group $G$ preserves the proper non-degenerate vector subspace $L^{\perp} \subset \mathbb{C}^{1, n+1}$.

Suppose that $\Gamma(G) \subset \operatorname{Sim} \mathbb{C}^{n}$ preserves a proper complex affine subspace $L \subset \mathbb{C}^{n}$. The subgroup $G \subset \mathrm{U}(1, n+1)_{\mathbb{C} p}$ is conjugated to a subgroup $G_{1} \subset \mathrm{U}(1, n+1)_{\mathbb{C} p}$ such that $\Gamma\left(G_{1}\right) \subset$ Sim $\mathbb{C}^{n}$ preserves a proper complex vector subspace corresponding to the affine subspace $L \subset \mathbb{C}^{n}$. This gives a contradiction, since it is clear that $G_{1} \subset \mathrm{U}(1, n+1)_{\mathbb{C} p}$ is weakly irreducible. This proves the proposition.

Let $\mathfrak{g} \subset \mathfrak{u}(1, n+1)_{\mathbb{C} p}$ be a weakly irreducible subalgebra. Let $G \subset \mathrm{U}(1, n+1)_{\mathbb{C} p}$ be the corresponding connected Lie subgroup. Suppose that $\Gamma(G)$ preserves a real affine subspace $L \subset \mathbb{C}^{n}$. Considering, if necessary, another group $G_{1}$ conjugated to $G$, we may assume that $L \subset \mathbb{C}^{n}$ is a real vector subspace. We may assume also that $L$ is minimal in the sense that $\Gamma(G)$ does not preserve any proper affine subspace in $L$. The induced action of $\Gamma(G)$ on the Euclidean space $L$ is by similarity transformations. Since $\Gamma(G)$ does not preserve any proper affine subspace of $L$, it acts transitively on $L[\mathbf{2}]$. Thus,

$$
\Gamma(G) \subset\left(\mathrm{O}\left(L^{\perp}\right) \cdot \operatorname{Sim} L\right) \cap \operatorname{Sim} \mathbb{C}^{n}=\left(\mathbb{R}^{*} \cdot\left(\left(\mathrm{O}\left(L^{\perp}\right) \cdot \mathrm{O}(L)\right) \cap \mathrm{U}(n)\right)\right) \wedge L,
$$

where the orthogonal complement is taken with respect to the Euclidean metric Re $h$, and

$$
\operatorname{Sim} L=\left(\mathbb{R}^{*} \cdot \mathrm{O}(L)\right) \curlywedge L
$$

is the Lie group of similarity transformations of the Euclidean space $L$. Moreover, the projection of $\Gamma(G)$ on $\operatorname{Sim} L$ is a transitive group of similarity transformations. The Lie algebras of the transitive Lie groups of similarity transformations of an Euclidean space $E$ are exhausted by the following [2]:

- $\mathfrak{f} \ltimes L$,

- $\left(\mathfrak{f}_{0} \oplus\{\psi(X)+X \mid X \in U\}\right) \ltimes W$,

where $\mathfrak{f} \subset \mathbb{R} \oplus \mathfrak{s o}(L)$ is a subalgebra; for the second algebra, there exists an orthogonal decomposition $L=W \oplus U, \mathfrak{f}_{0} \subset \mathfrak{s o}(W)$ is a subalgebra, $\psi: U \rightarrow \mathfrak{s o}(W)$ is a non-zero linear map such that $\mathfrak{f}_{0} \cap \psi(U)=0$ and $\psi(U)$ is commutative and it commutes with $\mathfrak{f}_{0}$.

Let us describe the subspace $L \subset \mathbb{C}^{n}$. It is clear that $i L \cap L \subset \mathbb{C}^{n}$ is a Hermitian subspace, let us denote it by $\mathbb{C}^{m}$. Let $\mathbb{C}^{n-m}$ denote the orthogonal complement to $\mathbb{C}^{m}$. We obtain the decomposition

$$
\mathbb{C}^{n}=\mathbb{C}^{m} \oplus \mathbb{C}^{n-m}
$$

Let $L_{0} \subset L$ be the orthogonal complement to $\mathbb{C}^{m} \subset L$ with respect to $\operatorname{Re} h$. It is clear that $i L_{0} \cap L_{0}=0$, i.e. $L_{0} \subset \mathbb{C}^{n-m}$ is a real form. We obtain the decomposition

$$
L=\mathbb{C}^{m} \oplus L_{0} .
$$

Let $e_{1}, \ldots, e_{m}$ be an $h$-orthonormal basis of $\mathbb{C}^{m}$. Consider a basis $f_{m+1}, \ldots, f_{n}$ of the real vector space $L_{0}$. We may assume that this basis is orthonormal with respect to Re $h$. There exists a skew-symmetric real matrix $\omega=\left(\omega_{j k}\right)_{j, k=m+1}^{n}$ such that

$$
h\left(f_{j}, f_{k}\right)=\delta_{j k}+i \omega_{j k}, \quad j, k=m+1, \ldots, n .
$$

It is known, that the basis $f_{m+1}, \ldots, f_{n}$ can be chosen in such a way that

$$
\omega=\operatorname{diag}\left(\left(\begin{array}{cc}
0 & -\lambda_{1} \\
\lambda_{1} & 0
\end{array}\right), \ldots,\left(\begin{array}{cc}
0 & -\lambda_{s} \\
\lambda_{s} & 0
\end{array}\right), 0, \ldots, 0\right)
$$

for some real numbers $\lambda_{k}$. Since $h$ is positive definite, it holds $\left|\lambda_{k}\right|<1$.

Let $\tau: \mathbb{C}^{n-m} \rightarrow \mathbb{C}^{n-m}$ be the anti-linear involution defining the real form $L_{0} \subset \mathbb{C}^{n-m}$. It holds

$$
\tau\left(f_{k}\right)=f_{k}, \quad \tau\left(i f_{k}\right)=-i f_{k}, \quad k=m+1, \ldots, n .
$$


Define the endomorphism $\theta$ of $L_{0}$ by the equation

$$
\operatorname{Re} h(\theta X, Y)=\operatorname{Im} h(X, Y), \quad X, Y \in L_{0}
$$

and extend it to a $\mathbb{C}$-linear endomorphism of $\mathbb{C}^{n-m}$. Clearly, $\theta \in \mathfrak{u}(n-m)$. Note that $\theta=0$ if and only if $L_{0}$ contains an $h$-orthonormal basis of $\mathbb{C}^{n-m}$.

For $k=1, \ldots, s$, let

$$
e_{m+2 k-1}=\frac{\sqrt{2}}{2 \sqrt{1-\lambda_{k}}}\left(f_{m+2 k-1}+i f_{m+2 k}\right), \quad e_{m+2 k}=\frac{\sqrt{2}}{2 \sqrt{1+\lambda_{k}}}\left(f_{m+2 k}+i f_{m+2 k-1}\right) .
$$

Then it holds

$$
\theta\left(e_{m+2 k-1}\right)=-i \lambda_{k} e_{m+2 k-1}, \quad \theta\left(e_{m+2 k}\right)=i \lambda_{k} e_{m+2 k}
$$

and

Note that

$$
\tau\left(e_{m+2 k-1}\right)=-i \frac{\sqrt{1+\lambda_{k}}}{\sqrt{1-\lambda_{k}}} e_{m+2 k}, \quad \tau\left(e_{m+2 k}\right)=-i \frac{\sqrt{1-\lambda_{k}}}{\sqrt{1+\lambda_{k}}} e_{m+2 k-1} .
$$

$$
\left(\mathfrak{s o}\left(L^{\perp}\right) \oplus \mathfrak{s o}(L)\right) \cap \mathfrak{u}(n)=\mathfrak{u}(m) \oplus \mathfrak{z} \mathfrak{u}(n-m) \tau,
$$

where $\mathfrak{z}_{\mathfrak{u}(n-m)} \tau$ denotes the ideal in $\mathfrak{u}(n-m)$ consisting of elements commuting with $\tau$.

Suppose that the projection of $\Gamma^{\prime}(\mathfrak{g})$ to $\mathfrak{s i m}(L)$ is of the form $\left(\mathfrak{f}_{0} \oplus\{\psi(X)+X \mid X \in U\}\right) \ltimes W$. Since $\mathfrak{f}=\mathfrak{f}_{0} \oplus \psi(U)$ annihilates $U$, and it is contained in $\mathfrak{u}(n)$, it annihilates also $i U$. Consequently, $\mathfrak{f}$ is contained in $\mathfrak{u}\left((U+i U)^{\perp}\right)$. We denote this space by $\mathbb{C}^{r}$. It is clear that $\mathbb{C}^{r} \subset L$. This implies that $\mathbb{C}^{r} \subset \mathbb{C}^{m}$. Let $\mathbb{C}^{m-r}$ be the orthogonal complement to $\mathbb{C}^{r}$ in $\mathbb{C}^{m}$. We get the decomposition

$$
L=\mathbb{C}^{r} \oplus \mathbb{C}^{m-r} \oplus L_{0}
$$

and we extend the map $\psi$ to $\mathbb{C}^{m-r} \oplus L_{0}$. Thus we may assume that $W=\mathbb{C}^{r}$, and $U=\mathbb{C}^{m-r} \oplus L_{0}$.

We conclude that if $\mathfrak{g} \subset \mathfrak{u}(1, n+1)_{\mathbb{C} p}$ is a weakly irreducible subalgebra with the associated subspace $L=\mathbb{C}^{m} \oplus L_{0} \subset \mathbb{C}^{n}$, then $\Gamma^{\prime}(\mathfrak{g})$ is one of the following:

- $\mathfrak{f} \ltimes L$,

- $\left(\mathfrak{f}_{0} \oplus\left\{\psi(X)+X \mid X \in \mathbb{C}^{m-r} \oplus L_{0}\right\}\right) \ltimes \mathbb{C}^{r}$,

where $\mathfrak{f} \subset \mathbb{R} \oplus \mathfrak{u}(m) \oplus \mathfrak{z} \mathfrak{u}(n-m) \tau$ is a subalgebra; $\mathfrak{f}_{0} \subset \mathfrak{u}(r), \psi: \mathbb{C}^{m-r} \oplus L_{0} \rightarrow \mathfrak{u}(r)$ is a non-zero linear map such that $\mathfrak{f}_{0} \cap \psi\left(\mathbb{C}^{m-r} \oplus L_{0}\right)=0$ and $\psi\left(\mathbb{C}^{m-r} \oplus L_{0}\right)$ is commutative and it commutes with $\mathfrak{f}_{0}$.

Let $\mathfrak{g}_{0}$ be the projection of $\mathfrak{g}$ to $\mathfrak{s u}(1, n+1)$. Note that if $m>0$, then from the structure of the Lie brackets in $\mathfrak{u}(1, n+1)_{\mathbb{C} p}$ it follows that $\mathfrak{g}_{0}$ contains the ideal $i \mathbb{R} \subset \mathfrak{u}(1, n+1)_{\mathbb{C} p}$. Even more, below we will see that all holonomy algebras $\mathfrak{g} \subset \mathfrak{u}(1, n+1)_{\mathbb{C} p}$ contain this ideal, thus it is enough to consider only such subalgebras. Then, since the kernel of $\left.\Gamma^{\prime}\right|_{\mathfrak{s u}(1, n+1)_{\mathbb{C} p}}$ coincides with $i \mathbb{R}$, we immediately find that

Note that

$$
\mathfrak{g}_{0}=\left(\left.\Gamma^{\prime}\right|_{\mathfrak{s u}(1, n+1)_{\mathbb{C} p}}\right)^{-1}\left(\Gamma^{\prime}(\mathfrak{g})\right)
$$

$$
\left(\left.\Gamma^{\prime}\right|_{\mathfrak{s u}(1, n+1)_{\mathbb{C} p}}\right)^{-1}\left(i \mathrm{id}_{\mathbb{C}^{m}}\right)=\frac{i}{n+2}\left(\begin{array}{cccc}
-m & 0 & 0 & 0 \\
0 & n+2-m & 0 & 0 \\
0 & 0 & -m & 0 \\
0 & 0 & 0 & -m
\end{array}\right)+i \mathbb{R} .
$$

Now, for the Lie algebra $\mathfrak{g}$ we have the following there possibilities:

$$
\mathfrak{g}=\mathfrak{g}_{0}, \quad \mathfrak{g}=\mathfrak{g}_{0} \oplus \mathbb{R} J, \quad \mathfrak{g}=\left\{A+\zeta(A) J \mid A \in \mathfrak{g}_{0}\right\},
$$

where $\zeta: \mathfrak{g}_{0} \rightarrow \mathbb{R}$ is a linear map zero on the commutant $\mathfrak{g}_{0}^{\prime}=\left[\mathfrak{g}_{0}, \mathfrak{g}_{0}\right]$.

Thus we conclude that if $\mathfrak{g} \subset \mathfrak{u}(1, n+1)_{\mathbb{C} p}$ is a weakly irreducible subalgebra containing the ideal $i \mathbb{R} \subset \mathfrak{u}(1, n+1)_{\mathbb{C} p}$, then $\mathfrak{g}$ is one of the following:

$$
\begin{aligned}
& \text { - } \mathfrak{f} \ltimes(L \ltimes i \mathbb{R}), \\
& \text { - }\left(\mathfrak{f}_{0} \oplus\left\{\psi(X)+X \mid X \in \mathbb{C}^{m-r} \oplus L_{0}\right\}\right) \ltimes\left(\mathbb{C}^{r} \ltimes i \mathbb{R}\right),
\end{aligned}
$$

where $\mathfrak{f} \subset \mathbb{R} \oplus \mathbb{R} J \oplus \mathfrak{u}(m) \oplus \mathfrak{z}_{\mathfrak{u}(n-m)} \tau$ is a subalgebra; $\mathfrak{f}_{0} \subset \mathfrak{u}(r) \oplus \mathbb{R} J, \psi: \mathbb{C}^{m-r} \oplus L_{0} \rightarrow \mathfrak{u}(r) \oplus \mathbb{R} J$ is a non-zero linear map such that $\mathfrak{f}_{0} \cap \psi\left(\mathbb{C}^{m-r} \oplus L_{0}\right)=0$ and $\psi\left(\mathbb{C}^{m-r} \oplus L_{0}\right)$ is commutative and it commutes with $\mathfrak{f}_{0}$. 


\section{Algebraic curvature tensors and Berger algebras}

Let $(M, g, J)$ be a Lorentz-Kähler manifold of complex dimension $n+2 \geq 2$, i.e. $g$ is a pseudo-Riemannian metric of signature $(2,2 n+2)$ on $M$, and $J$ is a parallel $g$-orthogonal complex structure. Consider the corresponding Lorentz-Kähler metric

$$
h=g+i g \circ J .
$$

Fix a point $x \in M$. Let $\mathfrak{g} \subset \mathfrak{u}\left(T_{x} M, h_{x}\right)$ be the holonomy algebra of the manifold $(M, g)$. We identify the tangent space $T_{x} M$ with the Lorentz-Hermitian space $\mathbb{C}^{1, n+1}$.

Consider the complexified tangent bundle $T^{\mathbb{C}} M$ and the standard decomposition

$$
T^{\mathbb{C}} M=T^{1,0} M \oplus T^{0,1} M
$$

into the direct sum of eigenspaces of the $\mathbb{C}$-linear extension of $J$ corresponding to the eigenvalues $\pm i$. Recall that sections of $T^{1,0} M$ and $T^{0,1} M$ are of the form

$$
X-i J X, \quad X+i J X,
$$

where $X$ is a vector field on $M$. In particular, the bundle $T^{1,0} M$ is identified with $T M$. The metric $g$ allows to identify the bundle $T^{0,1} M$ with the dual bundle to $T^{1,0} M$. Moreover, let $X, Y$ denote both vector fields in $T M$ and the corresponding vector fields in $T^{1,0} M$, then it holds

$$
g(X, \bar{Y})=4 h(X, Y)
$$

where $g$ denotes the $\mathbb{C}$-bilinear extension to $T^{\mathbb{C}} M$ of the initial metric $g$.

Fix a basis $p, e_{1}, \ldots, e_{n}, q$ in $T_{x} M$ as above. Denote by the same symbols $p, e_{1}, \ldots, e_{n}, q$ the basis

$$
\frac{1}{2}(p-i J p), \frac{1}{2}\left(e_{1}-i J e_{1}\right), \ldots, \frac{1}{2}\left(e_{n}-i J e_{n}\right), \frac{1}{2}(q-i J q) \in T_{x}^{1,0} M .
$$

Then the vectors $\bar{p}, \bar{e}_{1}, \ldots, \bar{e}_{n}, \bar{q}$ form a basis of $T_{x}^{0,1} M$. Suppose that $\mathfrak{g} \subset \mathfrak{u}(1, n+1)_{\mathbb{C} p}$. The holonomy algebra of the complex connection $\nabla$ coincides with the complexification $\mathfrak{g}^{\mathbb{C}}=\mathfrak{g} \otimes \mathbb{C}$ of $\mathfrak{g}$. The complexification of $\mathfrak{u}(1, n+1)_{\mathbb{C} p}$ consists of the matrices of the form

$$
\left(\begin{array}{cc}
T_{1} & 0 \\
0 & T_{2}
\end{array}\right)
$$

where

$$
T_{1}=\left(\begin{array}{ccc}
a & \bar{W}^{t} & c \\
0 & A & Z \\
0 & 0 & b
\end{array}\right), \quad T_{2}=-\left(\begin{array}{ccc}
b & \bar{Z}^{t} & c \\
0 & A^{t} & W \\
0 & 0 & a
\end{array}\right)
$$

with $a, b, c \in \mathbb{C}, Z, W \in \mathbb{C}^{n}$ and $A \in \mathfrak{g l}(n, \mathbb{C})$. Denote by $\mathfrak{g}^{1,0}$ the projection of $\mathfrak{g}^{\mathbb{C}}$ to $\mathfrak{g l}\left(T_{x}^{1,0} M\right)$. It is clear that this Lie algebra determines $\mathfrak{g}^{\mathbb{C}}$. This Lie algebra allows us also to determine $\mathfrak{g}$, namely, consider the following anti-linear involution of $\mathfrak{g}^{1,0}$ :

$$
\sigma:\left(\begin{array}{ccc}
a & \bar{W}^{t} & c \\
0 & A & Z \\
0 & 0 & b
\end{array}\right) \mapsto-\left(\begin{array}{ccc}
\bar{b} & \bar{Z}^{t} & \bar{c} \\
0 & \bar{A}^{t} & W \\
0 & 0 & \bar{a}
\end{array}\right) .
$$

Then $\mathfrak{g}$ coincides with the set of fixed points of $\sigma$.

The symbol $\nabla$ will denote both the Levi-Civita connection on $(M, g)$ and the corresponding complex connection on $T^{\mathbb{C}} M$. We use the same convection for $R$. It is well known that $R$ satisfies the conditions

$$
R(J X, J Y)=R(X, Y), \quad R(J X, Y)+R(X, J Y)=0
$$

for all vector fields on $M$. This implies

LEMMA 5.1. For all vector fields $Z, W \in T^{1,0} M$ it holds

$$
R(Z, W)=R(\bar{Z}, \bar{W})=0, \quad R(Z, \bar{W})=\overline{R(\bar{Z}, W)},
$$

where the conjugation is taken with respect to $\mathfrak{u}\left(T^{\mathbb{C}} M\right) \subset \mathfrak{g l}\left(T^{\mathbb{C}} M\right)$. In particular,

$$
R_{x}(Z, \bar{Z}) \in i \mathfrak{g} .
$$


It holds

$$
R(X, Y)=R(X, \bar{Y})-R(Y, \bar{X})
$$

where on the left hand side $R$ is the curvature tensor of the connection in $T M$ and $X, Y$ are vector fields on $X$, and on the right hand side $R$ is the curvature tensor of the complexified connection and $X, Y$ are vector fields from $T^{1,0} M$ corresponding to the initial $X, Y$.

Let now $\mathfrak{g} \subset \mathfrak{u}(1, n+1)_{\mathbb{C} p}$ be an arbitrary subalgebra. We may carry all the above notations to this case. Denote $\mathbb{C}^{1, n+1}$ simply by $V$, and as above we write

$$
V^{\mathbb{C}}=V \otimes \mathbb{C}=V^{1,0} \oplus V^{0,1}=V \oplus \bar{V} .
$$

For the Lie algebra $\mathfrak{g}^{\mathbb{C}}=\mathfrak{g} \otimes \mathbb{C}$ consider the following space of algebraic curvature tensors:

$$
\mathcal{R}\left(\mathfrak{g}^{\mathbb{C}}\right)=\left\{R: \Lambda^{2} V^{\mathbb{C}} \rightarrow \mathfrak{g}^{\mathbb{C}} \mid \begin{array}{c}
R(X, Y) Z+R(Y, Z) X+R(Z, X) Y=0, \forall X, Y, Z \in V^{\mathbb{C}} \\
R(X, \bar{Y})=\sigma(R(\bar{X}, Y)), \forall X, Y \in V
\end{array}\right\} .
$$

We say that $\mathfrak{g} \subset \mathfrak{u}(1, n+1) \mathbb{C} p$ is a Berger algebra if $\mathfrak{g}^{\mathbb{C}}$ is generated by the images of the elements $R$ : $\Lambda^{2} V^{\mathbb{C}} \rightarrow \mathfrak{g}^{\mathbb{C}}$ from the space $\mathcal{R}\left(\mathfrak{g}^{\mathbb{C}}\right)$. From the Ambrose-Singer theorem and the above consideration it follows that if $\mathfrak{g} \subset \mathfrak{u}(1, n+1)_{\mathbb{C} p}$ is the holonomy algebra of a Lorentz-Kähler manifold, then it is a Berger algebra.

The proof of the following lemma is direct.

Lemma 5.2. A linear map $R: \Lambda^{2} V^{\mathbb{C}} \rightarrow \mathfrak{g}^{\mathbb{C}}$ belongs to $\mathcal{R}\left(\mathfrak{g}^{\mathbb{C}}\right)$ if and only if it satisfies

$$
R(X, \bar{Y})=\sigma(R(\bar{X}, Y)), \quad R(X, \bar{Y}) Z=R(Z, \bar{Y}) X, \forall X, Y, Z \in V .
$$

For an element $\xi \in \mathfrak{g}^{\mathbb{C}}$ we denote by $\xi^{1,0}$ its projection onto $\mathfrak{g l}\left(V^{1,0}\right)=\mathfrak{g l}(V)$. The following lemma easily follows from the previous one. We denote by $X, Y$ vectors from $\mathbb{C}^{n}$.

LEMMA 5.3. Each algebraic curvature tensor $R \in \mathcal{R}\left(\mathfrak{u}(1, n+1)_{\mathbb{C} p} \otimes \mathbb{C}\right)$ is uniquely given by the equalities

$$
\begin{aligned}
& R^{1,0}(p, \bar{q})=\left(\begin{array}{ccc}
\alpha & N^{t} & \beta \\
0 & 0 & 0 \\
0 & 0 & 0
\end{array}\right), \quad R^{1,0}(X, \bar{q})=\left(\begin{array}{ccc}
g(N, X) & T(X)^{t} & g(\bar{K}, X) \\
0 & P(X) & A X \\
0 & 0 & 0
\end{array}\right), \\
& R^{1,0}(X, \bar{Y})=\left(\begin{array}{ccc}
0 & g(P(X) \cdot \bar{Y}) & g(A X, \bar{Y}) \\
0 & R_{0}(X, \bar{Y}) & \bar{P}^{t} X \\
0 & 0 & 0
\end{array}\right), \quad R^{1,0}(q, \bar{q})=\left(\begin{array}{ccc}
\beta & \bar{K}^{t} & c \\
0 & A & K \\
0 & 0 & \bar{\beta}
\end{array}\right)
\end{aligned}
$$

for arbitrary elements $\alpha, \beta, \in \mathbb{C}, c \in \mathbb{R}, N \in \overline{\mathbb{C}^{n}}, K \in \mathbb{C}^{n}, T \in \odot^{2} \overline{\mathbb{C}^{n}}, R_{0} \in \mathcal{R}\left(\mathfrak{u}(n)^{\mathbb{C}}\right), A \in \mathfrak{g l}(n, \mathbb{C})$ and $P \in \mathfrak{g l}(n, \mathbb{C})^{(1)}=\odot^{2} \overline{\mathbb{C}^{n}} \otimes \mathbb{C}^{n}$.

Now, for an arbitrary subalgebra $\mathfrak{g} \subset \mathfrak{u}(1, n+1)_{\mathbb{C} p}$ it holds

$$
\mathcal{R}\left(\mathfrak{g}^{\mathbb{C}}\right)=\mathcal{R}\left(\mathfrak{u}(1, n+1)_{\mathbb{C} p} \otimes \mathbb{C}\right) \cap\left(\Lambda^{2}\left(V^{\mathbb{C}}\right)^{*} \otimes \mathfrak{g}^{\mathbb{C}}\right) .
$$

Recall that we assign to each weakly irreducible subalgebra $\mathfrak{g} \subset \mathfrak{u}(1, n+1) \mathbb{C} p$ a real vector subspace $L=\mathbb{C}^{m} \oplus L_{0} \subset \mathbb{C}^{n}$ such that $L_{0} \subset \mathbb{C}^{n-m}$ is a real form.

THEOREM 5.1. Let $n \geq 1$. If $\mathfrak{g} \subset \mathfrak{u}(1, n+1) \mathbb{C} p$ is a weakly irreducible Berger subalgebra, then it is one of the following algebras:

$$
\begin{aligned}
\bullet & \mathfrak{g}^{\mathfrak{k}}=\mathfrak{k} \ltimes(L \ltimes i \mathbb{R}) \\
= & \left\{\left(\begin{array}{cccc}
a_{1}+i a_{2} & -\bar{Z}^{t} & -\bar{X}^{t} & i c \\
0 & A & 0 & Z \\
0 & 0 & a_{2}\left(i E_{n-m}+\theta\right) & X \\
0 & 0 & 0 & -a_{1}+i a_{2}
\end{array}\right) \mid \begin{array}{c}
a_{1}+a_{2}\left(i+i \mathrm{id}_{\mathbb{C}^{n-m}}+\theta\right)+A \in \mathfrak{k}, \\
Z \in \mathbb{C}^{m}, X \in L_{0}, \\
c \in \mathbb{R}
\end{array}\right. \\
\text { where } & k \in \mathbb{R} \oplus \mathbb{R}\left(i, i \operatorname{id}_{\mathbb{C}^{n-m}}+\theta\right) \oplus \mathfrak{u}(m)
\end{aligned}
$$

is an arbitrary subalgebra; 
- $\mathfrak{g}^{\mathfrak{k}, \psi}=\mathfrak{k} \oplus\left\{(0, \psi(X), X, 0) \mid X \in \mathbb{C}^{m-r} \oplus L_{0}\right\} \ltimes\left(\mathbb{C}^{r} \ltimes i \mathbb{R}\right)$

$$
=\left\{\left(\begin{array}{cccc}
0 & -\bar{Z}^{t} & -\bar{X}^{t} & i c \\
0 & A+\psi(X) & 0 & Z \\
0 & 0 & 0 & X \\
0 & 0 & 0 & 0
\end{array}\right) \mid \begin{array}{c}
A \in \mathfrak{k}, \\
Z \in \mathbb{C}^{r}, X \in \mathbb{C}^{m-r} \oplus L_{0}, \\
c \in \mathbb{R}
\end{array}\right\},
$$

where $1 \leq r \leq m$, we consider the decomposition

$$
\mathbb{C}^{m}=\mathbb{C}^{r} \oplus \mathbb{C}^{m-r}
$$

$\mathfrak{k} \subset \mathfrak{u}(r)$ is an arbitrary subalgebra,

$$
\psi: \mathbb{C}^{m-r} \oplus L_{0} \rightarrow \mathfrak{u}(r)
$$

is a non-zero linear map such that the image $\psi\left(\mathbb{C}^{m-r} \oplus L_{0}\right) \subset \mathfrak{u}(r)$ is commutative, it commutes with $\mathfrak{k}_{0}$ and has trivial intersection with $\mathfrak{k}_{0}$.

5.1. Proof of Theorem 5.1. We consider the Lie algebras obtained in Section 4 and verify, which of these Lie algebras are Berger algebras.

LEMMA 5.4. If $\mathfrak{g} \subset \mathfrak{u}(1, n+1)_{\mathbb{C} p}$ is a weakly irreducible Berger subalgebra contained in

$$
\mathbb{R} \oplus \mathbb{R}\left(i+i \operatorname{id}_{\mathbb{C}^{n-m}}\right) \oplus \mathfrak{u}(m) \oplus \mathfrak{z}_{\mathfrak{u}(n-m)} \tau \ltimes(L \ltimes i \mathbb{R}),
$$

then $\mathfrak{g}$ is contained in

$$
\mathbb{R} \oplus \mathbb{R}\left(i+i \mathrm{id}_{\mathbb{C}^{n-m}}+\theta\right) \oplus \mathfrak{u}(m) \ltimes(L \ltimes i \mathbb{R}) .
$$

Proof. Let $R \in \mathcal{R}\left(\mathfrak{g}^{\mathbb{C}}\right)$ be as in Lemma 5.3. It is clear that both $R_{0}$ and $P$ take their values in $\mathfrak{u}(m)$. Consider the tensor $A$, which should be of the form $A=i A_{1}+i A_{2}+a_{2} \operatorname{id}_{\mathbb{C}^{n-m}}$, where $A_{1} \in \mathfrak{u}(m), A_{2} \in \mathfrak{z}_{\mathfrak{u}(n-m)} \tau$, and $a_{2} \in \mathbb{R}$. Note that the complexification of the vector subspace $L \subset \mathfrak{g}$ has the form

$$
L \otimes \mathbb{C}=\left\{\left(\begin{array}{cccc}
0 & \bar{W}^{t} & -\overline{\tau(X)}^{t} & 0 \\
0 & 0 & 0 & Z \\
0 & 0 & 0 & X \\
0 & 0 & 0 & 0
\end{array}\right) \mid W, Z \in \mathbb{C}^{m}, X \in \mathbb{C}^{n-m}\right\}
$$

Let $X \in i L_{0}$, then this and the condition $R(X, \bar{q}) \in \mathfrak{g}^{\mathbb{C}}$ imply

$$
T(Y, X)=-g\left(Y, \overline{\tau\left(i A_{2} X+a_{2} X\right)}\right)
$$

for all $Y \in \mathbb{C}^{n}$. Since $X \in i L_{0}$ and $A_{2}$ preserves $i L$, it holds $\tau\left(i A_{2} X+a_{2} X\right)=i A_{2} X-a_{2} X$. Using (11) and the fact that $T$ is symmetric, we obtain

$$
h\left(Y, i A_{2} X-a_{2} X\right)=h\left(X, i A_{2} Y-a_{2} Y\right)
$$

for all $Y \in i L_{0}$. This implies

$$
\operatorname{Reh}\left(X, A_{2} Y\right)=a_{2} \operatorname{Im} h(X, Y)
$$

for all $X, Y \in L_{0}$, i.e. $A_{2}=-a_{2} \theta$. This proves the lemma.

LEMMA 5.5. If $\mathfrak{g} \subset \mathfrak{u}(1, n+1)_{\mathbb{C} p}$ is a weakly irreducible Berger subalgebra, then $i \mathbb{R} \subset \mathfrak{g}$.

Proof. Let $\mathfrak{g} \subset \mathfrak{u}(1, n+1)_{\mathbb{C} p}$ be a weakly irreducible Berger subalgebra and suppose that $i \mathbb{R} \not \subset \mathfrak{g}$. Then it is clear that $m=0$, i.e. $L=L_{0}$. By the previous lemma, $\operatorname{pr}_{\mathbb{C} \oplus \mathfrak{u}(n)} \mathfrak{g} \subset \mathbb{R}\left(i+i \operatorname{id}_{\mathbb{C}^{n-m}}+\theta\right)$. Let $R \in \mathcal{R}\left(\mathfrak{g}^{\mathbb{C}}\right)$ be as in Lemma 5.3 . From the condition that $R$ takes values in $\mathfrak{g}^{\mathbb{C}}$ it follows immediately that $R=0$.

LEMMA 5.6. If $\mathfrak{g} \subset \mathfrak{u}(1, n+1)_{\mathbb{C} p}$ is a weakly irreducible Berger subalgebra of the form $\left(\mathfrak{f}_{0} \oplus\right.$ $\left.\left\{\psi(X)+X \mid X \in \mathbb{C}^{m-r} \oplus L_{0}\right\}\right) \ltimes\left(\mathbb{C}^{r} \ltimes i \mathbb{R}\right)$, then $\mathfrak{f}_{0} \oplus \psi\left(\mathbb{C}^{m-r} \oplus L_{0}\right) \subset \mathfrak{u}(r)$. 
Proof. Suppose that $\operatorname{pr}_{\mathbb{C} \oplus \mathfrak{u}(n)} \mathfrak{g}$ contains an element of the form $i+i \mathrm{id}_{\mathbb{C}^{n-m}}+A$, where $A \in$ $\mathfrak{u}(r)$. Since $\mathfrak{g}$ is a Berger algebra, there exists $R \in \mathcal{R}\left(\mathfrak{g}^{\mathbb{C}}\right)$ such that

$$
R^{1,0}(q, \bar{q})=\left(\begin{array}{ccc}
1 & * & * \\
0 & \operatorname{id}_{\mathbb{C}^{n-m}}-i A & * \\
0 & 0 & 1
\end{array}\right) .
$$

Let $X \in \mathbb{C}^{m-r} \oplus L_{0}$. It holds

$$
R^{1,0}(X, \bar{q})=\left(\begin{array}{cccc}
0 & * & \operatorname{pr}_{\mathbb{C}^{m-r} \oplus L_{0}} T(X)^{t} & * \\
0 & 0 & 0 & * \\
0 & 0 & 0 & X \\
0 & 0 & 0 & 0
\end{array}\right) .
$$

This implies that $X-\operatorname{pr}_{\mathbb{C}^{m-r} \oplus L_{0}} \overline{T(X)} \in \mathfrak{g}$. Considering $i X$, we get $i X+i \operatorname{pr}_{\mathbb{C}^{m-r} \oplus L_{0}} \overline{T(X)} \in \mathfrak{g}$. The vectors of the last two types span $\mathbb{C}^{m-r} \oplus L_{0}$. We conclude that $\psi=0$. This gives a contradiction.

The lemmas show that if $\mathfrak{g} \subset \mathfrak{u}(1, n+1)_{\mathbb{C} p}$ is a weakly irreducible Berger subalgebra, then $\mathfrak{g}$ must be one of the algebras from the statement of the theorem. Conversely it is easy to see that all algebras from the statement of the theorem are weakly irreducible Berger algebras.

\section{Lorentz-Kähler metrics}

6.1. Complex Walker metrics. Suppose that $(M, g)$ is a Lorentzian manifold such that its holonomy group preserves an isotropic line, then $(M, g)$ admits a parallel distribution $\ell$ of isotropic lines. Locally there exist so called Walker coordinates $v, x^{1}, \ldots, x^{n}, u$ such that the metric $g$ has the form

$$
g=2 d v d u+h+2 A d u+H(d u)^{2},
$$

where $h=h_{i j}\left(x^{1}, \ldots, x^{n}, u\right) d x^{i} d x^{j}$ is an $u$-dependent family of Riemannian metrics, $A=A_{i}\left(x^{1}, \ldots, x^{n}, u\right) d x^{i}$ is an $u$-dependent family of one-forms, and $H=H\left(v, x^{1}, \ldots, x^{n}, u\right)$ is a local function on $M$ [16].

Let us show that Walker coordinates may be generalized to the case of a Lorentz-Kähler manifold. Suppose that $(M, h)$ is a Lorentzian-Kähler manifold such that its holonomy group preserves a complex isotropic line in the tangent space. Then $(M, h)$ admits a parallel distribution $\ell \subset T M$ of complex isotropic lines. It is clear that the corresponding line bundles $\ell \subset T^{1,0} M$ and $\bar{\ell} \subset T^{0,1} M$ are parallel. Moreover, the distribution $\ell^{\perp} \subset T M$ of complex codimension 1 is parallel, and the subbundles $\ell^{\perp} \subset T^{1,0} M$ and $\overline{\ell^{\perp}} \subset T^{0,1} M$ are parallel as well. It is clear that all parallel distributions are involutive. From the holomorphic version of the Frobenius Theorem it follows that locally on $M$ there exist holomorphic coordinates $v, z^{1}, \ldots, z^{n}, u$ such that the vector field $\partial_{v}$ generates the subbundle $\ell \subset T^{1,0} M$, and the vector fields $\partial_{v}, \partial_{z^{1}}, \ldots, \partial_{z^{n}}$ generate the subbundle $\ell^{\perp} \subset T^{1,0} M$. Consequently, the metric $h$ can be written in the following way:

$$
h=h_{\bar{u} v} d \bar{u} d v+h_{\bar{v} u} d \bar{v} d u+h_{\bar{j} k} d \bar{z}^{j} d z^{k}+h_{\bar{u} k} d \bar{u} d z^{k}+h_{\bar{k} u} d \bar{z}^{k} d u+h_{\bar{u} u} d \bar{u} d u .
$$

Since the metric $h$ is pseudo-Kähler, it holds

$$
\partial_{a} h_{\bar{b} c}=\partial_{c} h_{\bar{b} a}, \quad \partial_{\bar{a}} h_{\bar{b} c}=\partial_{\bar{b}} h_{\bar{a} c} .
$$

Hence the coefficients of the metric depend on the coordinates in the following way:

$$
\begin{gathered}
h_{\bar{v} u}=h_{\bar{v} u}\left(\bar{v}, \bar{z}^{l}, u, \bar{u}\right), \quad h_{\bar{j} k}=h_{\bar{j} k}\left(z^{l}, \bar{z}^{l}, u, \bar{u}\right), \\
h_{\bar{k} u}=h_{\bar{k} u}\left(\bar{v}, \bar{z}^{l}, u, \bar{u}\right), \quad h_{\bar{u} u}=h_{\bar{u} u}\left(v, \bar{v}, z^{l}, \bar{z}^{l}, u, \bar{u}\right)=h_{\bar{u} u}^{1}\left(v, z^{l}, u, \bar{u}\right)+h_{\bar{u} u}^{2}\left(\bar{v}, \bar{z}^{l}, u, \bar{u}\right)+h_{\bar{u} u}^{3}\left(z^{l}, \bar{z}^{l}, u, \bar{u}\right) .
\end{gathered}
$$

Thus any such metric is given by a potential $f$ that satisfies the equations

$$
\partial_{v} \partial_{\bar{v}} f=\partial_{v} \partial_{\bar{z}^{k}} f=0 .
$$

It is easy the check that the inverse matrix, defined by the equality

$$
h^{\bar{a} b} h_{\bar{a} c}=\delta_{c}^{b}
$$


is given by

$h^{\bar{v} v}=\frac{1}{\left|h_{\bar{u} v}\right|^{2}}\left(h_{\bar{l} u} h^{\bar{l} j} h_{\bar{u} j}-h_{\bar{u} u}\right), \quad h^{\bar{v} u}=\frac{1}{h_{\bar{v} u}}, \quad h^{\bar{j} k}=\tilde{h}^{\bar{j} k}, \quad h^{\bar{v} k}=-\frac{1}{h_{\bar{v} u}} h_{\bar{j} u} h^{\bar{j} k}, \quad h^{\bar{u} k}=h^{\bar{u} u}=0$, where $\tilde{h}^{\bar{j} k}$ is the inverse matrix to $h_{\bar{j} k}$.

For the obtained metric and $a \neq v$ it holds

$$
\Gamma_{v b}^{a}=h^{\bar{c} a} \partial_{b} h_{\bar{c} v}=h^{\bar{u} a} \partial_{b} h_{\bar{u} v}=0,
$$

i.e. the vector field $\partial_{v}$ is isotropic and recurrent, consequently it defines a local parallel distribution of complex isotropic lines $\ell \subset T^{1,0} M$.

We will consider the frame $p, e_{1}, \ldots, e_{n}, q, \bar{p}, \bar{e}_{1}, \ldots, \bar{e}_{n}, \bar{q}$, where

$$
p=\frac{1}{h_{\bar{v} u}} \partial_{v}, \quad e_{j}=C_{j}^{k}\left(\partial_{z^{k}}-\frac{h_{\bar{u} k}}{h_{\bar{u} v}} \partial_{v}\right), \quad q=\partial_{u}-\frac{h_{\bar{u} u}}{h_{\bar{u} v}} \partial_{v} .
$$

Here $C_{j}^{k}$ is a matrix such that $\bar{C}_{j}^{k} h_{\bar{k} l} C_{s}^{l}=\delta_{j l}$. It is clear that such matrix exists.

6.2. Non-existence result. Here we will show that some of the Berger algebras obtained above cannot appear as the holonomy algebras of Lorentz-Kähler metrics.

TheOREM 6.1. Let $\mathfrak{g}^{\mathfrak{k}}$ be the Berger algebra as in Theorem 5.1. If $m<n$ and $\mathfrak{g}^{\mathfrak{k}}$ is the holonomy algebra of a Lorentz-Kähler manifold, then $\mathfrak{k} \subset \mathbb{R}\left(i+i \operatorname{id}_{\mathbb{C}^{n-m}}\right) \oplus \mathfrak{u}(m)$. Consequently, if $\mathfrak{k} \not \subset \mathfrak{u}(m)$, then $\theta=0$, and $L_{0}=\mathbb{R}^{n-m}$.

Proof. Consider the Berger algebra $\mathfrak{g}^{\mathfrak{k}}$ as in Theorem 5.1 Suppose that $m<n$ and suppose that $\mathfrak{g}^{\mathfrak{k}} \otimes \mathbb{C}$ is the complexification of the holonomy algebra of a Lorentz-Kähler manifold $(M, h)$ at a point $x \in M$. Suppose that $\mathfrak{k} \otimes \mathbb{C}$ contains an element

$$
\xi=i a_{1}+i A+a_{2}(-\mathrm{id}+i \theta)
$$

where $A \in \mathfrak{u}(m)$. Suppose that $a_{1} \neq 0$, or $a_{2} \theta \neq 0$. Consider the coordinates and a local frame as in the previous section. Note that the holonomy algebra of the induced connection on the bundle $\ell^{\perp} / \ell$ coincides with $\operatorname{pr}_{\mathfrak{u}(n)} \mathfrak{k}$; this subbundle and its connection may be identified with the subbundle of $T^{1,0} M$ generated by the vector fields $e_{1}, \ldots, e_{n}$ and the connection $\nabla$ restricted and then projected to that subbundle. Let $\lambda$ be one of the non-zero eigenvalues of $i \theta$. Let $E_{\lambda x} \subset \operatorname{span}_{\mathbb{C}}\left\{e_{m+1}, \ldots, e_{n}\right\}$ be the eigenspace corresponding to the eigenvalue $-a_{2}(1-\lambda)$ of the operator $\xi$ restricted to $\operatorname{span}_{\mathbb{C}}\left\{e_{m+1}, \ldots, e_{n}\right\}$. The corresponding subspace in $\ell_{x}^{\perp} / \ell_{x}$ is clearly holonomy-invariant, and we obtain a parallel subbundle $E_{\lambda} \subset \ell^{\perp} / \ell$. Denote by the same symbol $E_{\lambda}$ the corresponding subbundle of $T^{1,0} M$, which is parallel modulo $p$. The holonomy algebra $\mathfrak{g}^{\mathfrak{k}} \otimes \mathbb{C}$ preserves the space of tensors of the form

$$
\bar{p}_{x} \wedge Z-p_{x} \wedge \overline{\kappa(Z)}
$$

where $Z \in E_{\lambda x}$ and $\kappa(Z)$ may be found from the condition

$$
h(\overline{\tau(X)}, Z)+h(\overline{\kappa(Z)}, X)=0, \quad \forall X \in \operatorname{span}_{\mathbb{C}}\left\{e_{1 x}, \ldots, e_{n x}\right\} .
$$

The element $\xi$ acts in this subspace as the multiplication by $a_{1} i+a_{2} \lambda$, and its orthogonal complement in the holonomy algebra acts in this space trivially. Consequently we get a parallel subbundle $F_{\lambda}$ of the bundle of bivectors. Let $y \in M$, and $\gamma$ be any curve from $x$ to $y$. Then $F_{\lambda y}=\tau_{\gamma} F_{\lambda x}$, and the element $\tau_{\gamma} \circ \xi \circ \tau_{\gamma}^{-1}$ of the holonomy algebra at the point $y$ acts on $F_{\lambda y}$ as the multiplication by $a_{1} i+a_{2} \lambda$. The same element acts on $\mathbb{C} p_{y} \wedge \bar{p}_{y}$ as the multiplication by $2 a_{1} i$. Consequently, $F_{\lambda}$ has trivial projection to the bundle $\langle p \wedge \bar{p}\rangle$ generated by $p \wedge \bar{p}$, and $F_{\lambda}$ consists of sections of the form

$$
\bar{p} \wedge Z-p \wedge \bar{W}
$$

where $Z \in \Gamma\left(E_{\gamma}\right)$ and $W$ is uniquely defined by $Z$. For each such section and each vector field $V$ from $T^{\mathbb{C}} M$ must hold

Hence,

$$
\operatorname{pr}_{<p \wedge \bar{p}>} \nabla_{V}(\bar{p} \wedge Z-p \wedge \bar{W})=0
$$

$$
\bar{p} \wedge \operatorname{pr}_{<p>} \nabla_{V} Z-p \wedge \operatorname{pr}_{<\bar{p}>} \nabla_{V} \bar{W}=0
$$


i.e.

Let $V=\partial_{z^{a}}$. Since $\bar{q}=\partial_{\bar{u}}-\frac{h_{\bar{u} u}}{h_{\bar{v} u}} \partial_{\bar{v}}$, it holds

$$
h\left(\nabla_{V} Z, \bar{q}\right)+h\left(\nabla_{V} \bar{W}, q\right)=0 .
$$

$$
h\left(\nabla_{\partial_{z^{a}}} Z, \bar{q}\right)=-h\left(Z, \nabla_{\partial_{z^{a}}} \bar{q}\right)=0 .
$$

Let $V=\partial_{\bar{z}^{a}}$. By the similar argument it holds $h\left(\nabla_{\partial \bar{z}^{a}} \bar{W}, q\right)=0$. Consequently,

$$
h\left(\nabla_{\partial \bar{z}^{a}} Z, \bar{q}\right)=0 .
$$

This shows that the subbundle $E_{\lambda} \subset T^{1,0} M$ is parallel. The corresponding distribution $E_{\lambda} \subset T M$ is also parallel, and it is non-degenerate. This gives a contradiction, since the holonomy algebra is weakly irreducible. Thus, $a_{1}=0$, and $a_{2} \theta=0$. This proves the theorem.

6.3. Construction of the metrics with all possible holonomy algebras. 1) First consider the complex dimension 2. Metrics with the holonomy algebra $\mathfrak{g}_{0}$ may be found in [7, 29.

Let $a, b \in \mathbb{C}$ be some numbers. We assume that if $a=0$, then $b=0$. If $a=b=0$, then we set

$$
f_{\mathbb{C}}=\bar{v} u+\bar{u} v
$$

if $a \neq 0, b=0$, we set

$$
f_{\mathbb{C}}(v, \bar{v}, u, \bar{u})= \begin{cases}2 \operatorname{Re}\left(-\frac{v}{i a u}\left(e^{-i a|u|^{2}}-1\right)\right), & \text { if } u \neq 0 \\ 0, & \text { if } u=0 .\end{cases}
$$

We claim that the function $f_{\mathbb{C}}$ is real analytic. Indeed, the function

$$
F(z)=e^{-i a z}-1
$$

is holomorphic and it vanishes at the point $z=0$. This implies that there exists an holomorphic function $H(z)$ such that

Consequently,

$$
F(z)=z H(z)
$$

$$
\frac{1}{u}\left(e^{-i a|u|^{2}}-1\right)=\bar{u} H\left(|u|^{2}\right) .
$$

This shows that the function $f_{\mathbb{C}}$ is real analytic. Note that it holds

$$
\partial_{\bar{u}} \partial_{v} f_{\mathbb{C}}=e^{-i a|u|^{2}} .
$$

Next, if $a, b \neq 0$, then let

$$
f_{\mathbb{C}}(v, \bar{v}, u, \bar{u})= \begin{cases}2 \operatorname{Re}\left(e^{\frac{i a^{2}}{b}} \frac{\sqrt{\pi} v}{\sqrt{i b} u}\left(\operatorname{erf}\left(\frac{i a}{\sqrt{i b}}+\frac{\sqrt{i b}|u|^{2}}{2}\right)-\operatorname{erf}\left(\frac{i a}{\sqrt{i b}}\right)\right)\right), & \text { if } u \neq 0 \\ 0, & \text { if } u=0\end{cases}
$$

Recall that the Error function $\operatorname{erf}(z)$ satisfies

$$
\frac{d}{d z} \operatorname{erf}(z)=\frac{2}{\sqrt{\pi}} e^{-z^{2}}
$$

The function $f_{\mathbb{C}}$ is again real analytic and it satisfies

$$
\partial_{\bar{u}} \partial_{v} f_{\mathbb{C}}=e^{-i a|u|^{2}-\frac{i b}{4}|u|^{4}} .
$$

The function $f_{\mathbb{C}}$ will be a part of the potential $f$ that we are going to construct; this function is chosen in such a way that the Lorentz-Kähler matric for the potential $f$ satisfies the condition

$$
h_{\bar{u} v}=e^{-i a|u|^{2}-\frac{i b}{4}|u|^{4}} .
$$

We could define the metric $h$ directly without considering its potential, then we could avoid the usage of the Error function and functions with $u$ in denominator, but then a priori the metric would be only pseudo-Hermitian and we would have to check the condition (3) in order to show that the metric is pseudo-Kähler.

Let $\mathfrak{g}$ denote the holonomy algebra of a metric given by some potential $f$. Similarly as in the proof of Theorem 6.2 the following may be shown:

if we chose $f=f_{\mathbb{C}}$ with $a=i, b=1$, then $\mathfrak{g}=\mathfrak{g}_{1}$; 
if we choose $f=f_{\mathbb{C}}$ with $a=\gamma \neq 0, b=0$, then $\mathfrak{g}=\mathfrak{g}_{3}^{\gamma}$;

if we choose $f=\bar{u} v+\bar{v} u+|u|^{4}$, then $\mathfrak{g}=\mathfrak{g}_{3}^{0}$;

finally, the holonomy algebra of the metric $h=e^{\bar{u} v} d \bar{u} d v+e^{\bar{v} u} d \bar{v} d u$ coincides with $\mathfrak{g}_{2}$.

2) Let $n \geq 1$. Denote by $v, z^{1}, \ldots, z^{n}, u$ the coordinates on $\mathbb{C}^{n+2}$. Let $\mathfrak{k} \subset \mathbb{C} \oplus \mathfrak{u}(n)$ be a subalgebra. Let us choose the following elements spanning $\mathfrak{k}$ :

$$
a+A_{1}, \quad b+A_{2}, \quad A_{3}, \ldots, A_{N},
$$

where $a, b \in \mathbb{C}, A_{1}, \ldots, A_{N} \in \mathfrak{u}(n)$. We may assume the following: if $a=0$, then $b=0$; if $a \neq 0$ (resp. $b \neq 0$ ), then $A_{1}$ (resp. $A_{2}$ ) belongs to the center of $\operatorname{pr}_{\mathfrak{u}(n)} \mathfrak{k}$.

Consider the following Hermitian matrices:

$$
B_{\alpha}=-\frac{1}{(\alpha !)^{2}} i A_{\alpha}, \alpha=1, \ldots, N
$$

and

$$
G=\sum_{\alpha=1}^{N} B_{\alpha}|u|^{2 \alpha}
$$

Define the function

$$
f_{\mathfrak{u}(n)}=\bar{Z}^{T} e^{G} Z
$$

where $e^{G}$ is the matrix exponential and $Z=\left(\begin{array}{c}z^{1} \\ \vdots \\ z^{n}\end{array}\right)$.

We may suppose that the basis $e_{1}, \ldots, e_{n}$ is chosen in such a way that, for some $0 \leq n_{0} \leq$ $m, A_{1}$ restricted to $\mathbb{C}^{n_{0}}=\operatorname{span}_{\mathbb{C}}\left\{e_{1}, \ldots, e_{n_{0}}\right\}$ is an isomorphism of $\mathbb{C}^{n_{0}}$, and it annihilates $\operatorname{span}_{\mathbb{C}}\left\{e_{n_{0}}, \ldots, e_{n}\right\}$. Define the following function:

$$
f_{\mathbb{C}^{m}}=\frac{1}{2} \operatorname{Re}\left(i \bar{u}^{2} \sum_{k=n_{0}+1}^{m}\left(z^{k}\right)^{2}\right) .
$$

Define the function

$$
f_{1}=f_{\mathbb{C}}+f_{\mathfrak{u}(n)}+f_{\mathbb{C}^{n}} .
$$

Consider the Lie algebra $\mathfrak{g}^{\mathfrak{k}, J, L}$. We assume that $a=i, b=0$,

$$
A_{1}=\tilde{A}_{1}+i \operatorname{id}_{\mathbb{C}^{n-m}},
$$

where $\tilde{A}_{1} \in u(m)$, and $A_{2}, \ldots, A_{N} \in \mathfrak{u}(m)$. Let $n_{0}$ be the number defined by $\tilde{A}_{1}$ in the same way as it was defined above by $A_{1}$. Let

$$
f_{\mathbb{R}^{n-m}}\left(z^{l}, \bar{z}^{l}, u, \bar{u}\right)= \begin{cases}-\operatorname{Re}\left(\sum_{j=m+1}^{n}\left(\bar{z}^{j}\right)^{2} \frac{1}{\bar{u}^{2}}\left(1-e^{|u|^{2}}+|u|^{2} e^{|u|^{2}}\right)\right), & \text { if } u \neq 0 ; \\ 0, & \text { if } u=0 .\end{cases}
$$

Note that the function

$$
F(z)=1-e^{z}+z e^{z}
$$

is holomorphic and it satisfies $F(0)=\frac{d}{d z} F(0)=0$. This implies that there exists a holomorphic function $G(z)$ such that $F(z)=z^{2} G(z)$. Consequently the function $f_{\mathbb{R}^{n-m}}$ is real analytic. It holds

$$
\partial_{\bar{z}^{j}} \partial_{u} f_{\mathbb{R}^{n-m}}=-\bar{z}^{j} u e^{|u|^{2}}, \quad j=m+1, \ldots, n .
$$

Define the function

$$
f_{2}=f_{\mathbb{C}}+f_{\mathfrak{u}(n)}+f_{\mathbb{C}^{m}}+f_{\mathbb{R}^{n-m}} .
$$

Consider the Lie algebra $\mathfrak{g}^{\mathfrak{k}, L}$. We assume that $a=b=0, A_{1}, \ldots, A_{N} \in \mathfrak{u}(m)$. Using the real form $L_{0} \subset \mathbb{C}^{n-m}$, we define the matrix

$$
B=\left(B_{k j}\right)_{k, j=m+1}^{n}=\operatorname{diag}\left(\frac{\sqrt{2}}{2}\left(\begin{array}{cc}
\sqrt{1-\lambda_{1}} & \sqrt{1+\lambda_{1}} \\
-i \sqrt{1+\lambda_{1}} & -i \sqrt{1-\lambda_{1}}
\end{array}\right), \ldots, \frac{\sqrt{2}}{2}\left(\begin{array}{cc}
\sqrt{1-\lambda_{s}} & \sqrt{1+\lambda_{s}} \\
-i \sqrt{1+\lambda_{s}} & -i \sqrt{1-\lambda_{s}}
\end{array}\right), 1, \ldots, 1\right) .
$$


Let

$$
f_{L_{0}}=-2 \operatorname{Re}\left(\sum_{j=m+1}^{n} \sum_{\alpha=1}^{n-m} \frac{i B_{j m+\alpha} \bar{z}^{j}}{((N+\alpha) !)^{2}}|u|^{2(N+\alpha)} \frac{u}{N+\alpha+1}\right)
$$

and

$$
f_{3}=f_{\mathbb{C}}+f_{\mathfrak{u}(n)}+f_{\mathbb{C}^{m}}+f_{L_{0}} .
$$

Let us turn to the algebra $\mathfrak{g}^{\mathfrak{k}_{0}, \psi}$. Let $\mathfrak{k}=\mathfrak{k}_{0} \oplus \psi\left(\mathbb{C}^{m-r} \oplus L_{0}\right)$. Consider the unitary matrices

$$
\begin{gathered}
A_{1}=\psi\left(e_{r+1}\right), \ldots, A_{m-r}=\psi\left(e_{m}\right), \quad A_{m-r+1}=\psi\left(i e_{r+1}\right), \ldots, A_{2 m-2 r}=\psi\left(i e_{m}\right), \\
A_{2 m-2 r+1}=\psi\left(f_{m+1}\right), \ldots, A_{n+m-2 r}=\psi\left(f_{n}\right),
\end{gathered}
$$

where, as above, $f_{m+1}, \ldots, f_{n}$ is a basis of the real vector space $L_{0}$. Let $A_{n+m-2 r+2}, \ldots, A_{N}$ be a basis of $\mathfrak{k}_{0}$. Let

$$
D=\left(\begin{array}{ccc}
i E_{m-r} & -E_{m-r} & 0 \\
0 & 0 & i B
\end{array}\right)
$$

Using this matrix, we define the function

$$
f_{\psi}=-\operatorname{Re}\left(\sum_{j=r+1}^{n} \sum_{\alpha=1}^{n+m-2 r} \frac{D_{j \alpha}\left(\bar{z}^{j}\right)^{2}}{((\alpha+2) !)^{2}}|u|^{2(\alpha+2)} \frac{u}{\alpha+3}\right) .
$$

Finally consider the function

$$
f_{4}=f_{\mathbb{C}}+f_{\mathfrak{u}(n)}+f_{\mathbb{C}^{r}}+f_{\psi} .
$$

TheOREM 6.2. The holonomy algebras of the Lorentz-Kähler metrics with the potentials $f_{1}$, $f_{2}, f_{3}, f_{4}$ coincide respectively with the Lie algebras $\mathfrak{g}^{\mathfrak{k}}, \mathfrak{g}^{\mathfrak{k}, J, L}, \mathfrak{g}^{\mathfrak{k}, L}, \mathfrak{g}^{\mathfrak{k}_{0}, \psi}$.

6.4. Proof of Theorem 6.2. Let $(M, h)$ be a Lorentz-Kähler manifold, let $\mathfrak{g} \subset \mathfrak{u}\left(T_{x} M\right)$ be the holonomy algebra of the Levi-Civita connection on $(M, h)$. Recall that the complexification $\mathfrak{g} \otimes \mathbb{C} \subset \mathfrak{g l}\left(T_{x}^{\mathbb{C}} M\right)$ coincides with holonomy algebra of the induced complex connection on $T^{\mathbb{C}} M$, and it is determined by the projection of $\mathfrak{g} \otimes \mathbb{C}$ to $\mathfrak{g l}\left(T_{x}^{1,0} M\right)$. If the pseudo-Riemannian metric corresponding to $h$ is analytic, then the holonomy algebra $\mathfrak{g} \otimes \mathbb{C}$ is generated by the following elements:

$$
\nabla_{Z_{r}} \cdots \nabla_{Z_{1}} R_{x}(X, \bar{Y}), \quad r \geq 0, X, Y \in T_{x}^{1,0} M, Z_{1}, \ldots, Z_{r} \in T_{x}^{\mathbb{C}} M .
$$

Let $z^{a}$ be local complex coordinates on $M$. We denote by $R^{1,0}\left(\partial_{z^{a}}, \partial_{\bar{z}^{b}}\right)$ the matrix of the field of the endomorphisms $R\left(\partial_{z^{a}}, \partial_{\bar{z}^{b}}\right)$ restricted to $T^{1,0} M$. Recall that the Christoffel symbols $\Gamma_{B C}^{A}$ are given by the equality

$$
\nabla_{\partial_{z_{B}}} \partial_{z^{A}}=\Gamma_{C B}^{A} \partial_{z^{C}}
$$

where we assume that $A, B, C$ take all values $a$ and $\bar{a}$. The possibly non-zero symbols are $\Gamma_{b c}^{a}$ and $\Gamma_{\bar{b} \bar{c}}^{\bar{a}}=\overline{\Gamma_{b c}^{a}}$. It holds

$$
\Gamma_{b c}^{a}=h^{\bar{d} a} \partial_{c} h_{\bar{d} b} .
$$

The components of the curvature tensor are defined by the equality

$$
R\left(\partial_{z^{C}}, \partial_{z^{D}}\right) \partial_{z^{B}}=R_{B C D}^{A} \partial_{z^{A}} .
$$

Only the following coefficients may be non-zero:

$$
R_{b c \bar{d}}^{a}=-R_{b \bar{d} c}^{a}, \quad-R_{\bar{b} d \bar{c}}^{\bar{a}}=R_{\bar{b} \bar{c} d}^{\bar{a}}=\overline{R_{b c \bar{d}}^{a}},
$$

and it holds

$$
R_{b c \bar{d}}^{a}=-\partial_{\bar{z}^{d}} \Gamma_{b c}^{a} .
$$

Let for each fixed $c, \Gamma_{c}$ denote the matrix $\left(\Gamma_{b c}^{a}\right)$. Let $\xi$ be the matrix of a one of the fields

$$
\nabla_{\partial_{z^{A}}} \cdots \nabla_{\partial_{z^{A_{1}}}} R^{1,0}\left(\partial_{z^{a}}, \partial_{\bar{z}^{b}}\right) \text {. }
$$

It holds

$$
\nabla_{\partial_{z^{c}}} \xi=\partial_{z^{c}} \xi+\left[\Gamma_{c}, \xi\right], \quad \nabla_{\partial_{\bar{z}} c} \xi=\partial_{\bar{z}^{c}} \xi
$$


Let now $h$ be the metric defined by a potential $f$ from the theorem. The coefficients of the metric $h$ are analytic as complex-valued functions of real variables, i.e. the holonomy algebra $\mathfrak{g} \otimes \mathbb{C}$ of the metric is generated by the elements (4).

It holds

$$
h_{\bar{u} v}=e^{-i a|u|^{2}-\frac{i b}{4}|u|^{4}}, \quad h_{\bar{j} k}=\left(e^{G}\right)_{\bar{j} k} .
$$

We will consider the frame $p, e_{1}, \ldots, e_{n}, q, \bar{p}, \bar{e}_{1}, \ldots, \bar{e}_{n}, \bar{q}$, where

$$
p=\frac{1}{h_{\bar{u} v}} \partial_{v}, \quad e_{j}=\left(e^{-\frac{1}{2} \bar{G}}\right)_{k j}\left(\partial_{z^{k}}-\frac{h_{\bar{u} k}}{h_{\bar{u} v}} \partial_{v}\right), \quad q=\partial_{u}-\frac{h_{\bar{u} u}}{h_{\bar{u} v}} \partial_{v} .
$$

Using this frame, the curvature tensor of the metric $h$ can be expressed through some tensor fields in the same way as in Section 5 (we will use the same notations). Note that

$$
\Gamma_{v v}^{v}=\Gamma_{v k}^{v}=\Gamma_{j k}^{l}=0
$$

Consequently, $\alpha=0, N=0, P=0$, and $R_{0}=0$.

Next,

and

$$
\Gamma_{u u}^{k}=h^{\bar{l} k} h_{\bar{v} u} \partial_{u}\left(\frac{h_{\bar{l} u}}{h_{\bar{v} u}}\right)
$$

$K=\operatorname{pr}_{<e_{1}, \ldots, e_{n}>} R(q, \bar{q}) q=\operatorname{pr}_{<e_{1}, \ldots, e_{n}>} R\left(\partial_{u}, \partial_{\bar{u}}\right) \partial_{u}$

$$
=\operatorname{pr}_{<e_{1}, \ldots, e_{n}>} R_{u u \bar{u}}^{k} \partial_{z^{k}}=\sum_{j=1}^{n}\left(e^{\frac{1}{2} \bar{G}}\right)_{\bar{j} k} R_{u u \bar{u}}^{k} e_{j} .
$$

Consequently,

$$
K=-\sum_{k, j=1}^{n}\left(e^{\frac{1}{2} \bar{G}}\right)_{\bar{j} k} \partial_{\bar{u}}\left(\left(e^{-G}\right)_{\bar{l} k} h_{\bar{v} u} \partial_{u}\left(\frac{h_{\bar{l} u}}{h_{\bar{v} u}}\right)\right) e_{j}
$$

Similarly,

$$
T\left(e_{j}\right)=-\sum_{k, k_{1}, j_{1}=1}^{n}\left(e^{-\frac{1}{2} \bar{G}}\right)_{\bar{j}_{1 j}}\left(e^{-\frac{1}{2} \bar{G}}\right)_{\bar{k}_{1} k} h_{\bar{u} v} \partial_{\bar{u}}\left(\frac{1}{h_{\bar{u} v}} \partial_{z^{j}} h_{\bar{u} k_{1}}\right) \bar{e}_{k} .
$$

Let $\mathfrak{g} \subset \mathfrak{u}(1, n+1)$ be the holonomy algebra of the metric $h$ at the point 0 .

LEMMA 6.1. It holds $\operatorname{pr}_{\mathbb{C} \oplus \mathfrak{u}(n)} \mathfrak{g}=\mathfrak{k}$.

Proof.

It is easy to check that

and

$$
\Gamma_{v u}^{v}=-i a \bar{u}-\frac{i b}{2}|u|^{2} \bar{u}
$$

$$
R_{p}^{p}(q, \bar{q})=i a+i b|u|^{2}
$$

Consequently, a non-trivial value at the point 0 at the position $(v, v)$ may have only the matrices of the following covariant derivative of $R$ :

$$
\nabla_{\partial_{u}} \nabla_{\partial_{\bar{u}}} R_{v u \bar{u}}^{v}(0)=\nabla_{\partial_{\bar{u}}} \nabla_{\partial_{u}} R_{v u \bar{u}}^{v}(0)=b i .
$$

Consider the matrix $\tilde{\Gamma}_{u}=\left(\Gamma_{j u}^{k}\right)_{j, k=1}^{n}$. Let $\tilde{R}(X, \bar{Y})$ be the similar matrix obtained from $R$ and vector fields $X, Y \in T^{1,0} M$. Let $X, Y \in\left\{p, e_{1}, \ldots, e_{n}, q\right\}$, then the last matrix $\tilde{R}(X, \bar{Y})$ is non-zero only for $X=Y=q$. Moreover,

$$
\tilde{R}(q, \bar{q})=\tilde{R}\left(\partial_{u}, \partial_{\bar{u}}\right)
$$

It holds

$$
\tilde{\Gamma}_{u}=e^{-G} \partial_{u} e^{G} .
$$

The formula for the derivation of the matrix exponential reads [43, Sec. 5, Th. 1.2]:

$$
e^{-G} \partial_{u} e^{G}=\sum_{k=0}^{\infty} \frac{(-1)^{k}}{(k+1) !}\left(\operatorname{ad}_{G}\right)^{k} \partial_{u} G=\partial_{u} G-\frac{1}{2 !}\left[G, \partial_{u} G\right]+\frac{1}{3 !}\left[G,\left[G, \partial_{u} G\right]\right]-\cdots
$$


Next,

LEMma 6.2. It holds

$$
\tilde{R}(q, \bar{q})=-\partial_{\bar{u}} \tilde{\Gamma}_{u}
$$

$$
\nabla_{\partial_{u}}^{r} \nabla_{\partial_{\bar{u}}}^{r} \tilde{R}\left(\partial_{u}, \partial_{\bar{u}}\right)(0)=i A_{r}, \quad r=0, \ldots, N
$$

Proof. From (5) it follows that

$$
\nabla_{\partial_{\bar{u}}}^{r} \tilde{R}\left(\partial_{u}, \partial_{\bar{u}}\right)=-\partial_{\bar{u}}^{r+1} \tilde{\Gamma}_{u}=\frac{1}{r !} i A_{r} u^{r}-\frac{1}{2 !} \partial_{\bar{u}}^{r+1}\left[G, \partial_{u} G\right]+\frac{1}{3 !} \partial_{\bar{u}}^{r+1}\left[G,\left[G, \partial_{u} G\right]\right]-\cdots
$$

Note that the covariant derivative with respect to $\partial_{u}$ contains the Lie brackets with the matrix $\tilde{\Gamma}_{u}$. On the other hand, $\tilde{\Gamma}_{u}$ is dividable by $\bar{u}$, consequently

$$
\begin{aligned}
\nabla_{\partial_{u}}^{r} \nabla_{\partial_{\bar{u}}}^{r} \tilde{R}\left(\partial_{u}, \partial_{\bar{u}}\right)(0)=\partial_{u}^{r} \partial_{\bar{u}}^{r} \tilde{R}\left(\partial_{u}, \partial_{\bar{u}}\right)(0) \\
\quad=i A_{r}-\frac{1}{2 !} \partial_{\bar{u}}^{r+1} \partial_{u}^{r}\left[G, \partial_{u} G\right](0)+\frac{1}{3 !} \partial_{\bar{u}}^{r+1} \partial_{u}^{r}\left[G,\left[G, \partial_{u} G\right]\right](0)-\cdots .
\end{aligned}
$$

Note that since all terms in $G$ contain the same powers of $u$ and $\bar{u}$, it holds

$$
\partial_{\bar{u}}^{r+1} \partial_{u}^{r}\left[G, \partial_{u} G\right](0)=\sum_{\substack{r_{1}+r_{2}=r+1 \\ r_{1} \geq 0, r_{2} \geq 1}}\left[\partial_{\bar{u}}^{r_{1}} \partial_{u}^{r_{1}} G, \partial_{\bar{u}}^{r_{2}} \partial_{u}^{r_{2}} G\right](0)=\left[G, \partial_{\bar{u}}^{r+1} \partial_{u}^{r+1} G\right](0)=0 .
$$

Here we used the symmetry in $r_{1}, r_{2}$ combined with the skew-symmetry of the Lie brackets, and the fact that $G(0)=0$. By the same reason $\partial_{\bar{u}}^{r+1} \partial_{u}^{r}\left[G,\left[G, \partial_{u} G\right]\right](0)$ as well as all terms containing more Lie brackets with $G$, are zero.

Note that, in particular,

$$
\tilde{R}\left(\partial_{u}, \partial_{\bar{u}}\right)(0)=i A_{1}, \quad \nabla_{\partial_{u}} \nabla_{\partial_{\bar{u}}} \tilde{R}\left(\partial_{u}, \partial_{\bar{u}}\right)(0)=\nabla_{\partial_{\bar{u}}} \nabla_{\partial_{u}} \tilde{R}\left(\partial_{u}, \partial_{\bar{u}}\right)(0)=i A_{2} .
$$

The elements $A_{1}$ and $A_{2}$ are in the center of $\operatorname{pr}_{\mathfrak{u}(n)} \mathfrak{k}$, consequently, by the construction, the other covariant derivatives of $\tilde{R}$ at the point 0 take values in the subalgebra of $\operatorname{pr}_{\mathfrak{g r}(n, \mathbb{C})} \mathfrak{k}$ orthogonal to $A_{1}$ and $A_{2}$. Using this and comparing the last equalities with (6), we conclude that $\mathfrak{k} \subset \operatorname{pr}_{\mathbb{C} \oplus \mathfrak{u}(n)} \mathfrak{g}$. The inverse inclusion easily follows from the construction and the above formulas. Lemma 6.1 is proved.

Let now $f=f_{1}$.

Lemma 6.3. It holds $\mathbb{C}^{n} \ltimes i \mathbb{R} \subset \mathfrak{g}$.

Proof. We know already that each holonomy algebra contains $i \mathbb{R}$. It holds

$$
R^{1,0}\left(e_{j}, \bar{q}\right)(0)=\left(\begin{array}{ccc}
0 & T\left(e_{j}\right)^{t} & * \\
0 & 0 & A_{1} e_{j} \\
0 & 0 & 0
\end{array}\right) \in \mathfrak{g}^{1,0}
$$

Next, $T\left(e_{j}\right)=0$ for $j=1, \ldots, n_{0}$, and $T\left(e_{j}\right)=-i \bar{e}_{j}$ for $j=n_{0}+1, \ldots, n$. This shows that $\mathfrak{g}$ contains $A_{1} e_{j}, i A_{1} e_{j} \in \mathbb{C}^{n}$ for $j=1, \ldots, n_{0}$. From the definition of $n_{0}$ it follows that $\mathfrak{g}$ contains $\mathbb{C}^{n_{0}}$. For each $j>n_{0}, \mathfrak{g}$ contains $e_{j}$ and $i e_{j}$. We conclude that $\mathfrak{g}$ contains $\mathbb{C}^{n} \ltimes i \mathbb{R}$.

From Lemmas 6.1 and 6.3 it follows that $\mathfrak{g}=\mathfrak{g}^{\mathfrak{k}}$.

Suppose that $f=f_{2}$.

Lemma 6.4. It holds $\operatorname{pr}_{\mathbb{C}^{n} \ltimes i \mathbb{R}} \mathfrak{g}=L \ltimes i \mathbb{R}=\mathbb{C}^{m} \oplus \mathbb{R}^{n-m} \ltimes i \mathbb{R} \subset \mathfrak{g}$.

Proof. Similarly as in Lemma 6.3 it can be shown that $\mathbb{C}^{m} \ltimes i \mathbb{R} \subset \mathfrak{g}$. Let $j \geq m+1$. It holds

$$
R\left(e_{j}, \bar{q}\right)=\left(\begin{array}{cccc}
0 & * & \bar{e}_{j}^{t} & * \\
0 & 0 & 0 & * \\
0 & 0 & 0 & -e_{j} \\
0 & 0 & 0 & 0
\end{array}\right)
$$

Consequently, $\mathfrak{g}$ contains $\mathbb{R}^{n-m}$. The covariant derivatives of this tensor give trivial projections on $\mathbb{C}^{n-m}$. Finally, $K=0$, i.e.

$$
R^{1,0}(q, \bar{q})=-\operatorname{id}_{T^{1,0} M}+c p \wedge \bar{p}
$$


for some function $c$. Hence, the covariant derivatives of $R^{1,0}(q, \bar{q})$ give trivial projections on $\mathbb{C}^{n-m}$.

From Lemmas 6.1 and 6.4 it follows that $\mathfrak{g}=\mathfrak{g}^{\mathfrak{k}, J, L}$.

Suppose that $f=f_{3}$. In this case $\mathfrak{k} \subset \mathfrak{u}(m)$. As above it can be shown that $\mathbb{C}^{m} \ltimes i \mathbb{R} \subset \mathfrak{g}$. It holds

$$
\operatorname{pr}_{<e_{m+1}, \ldots, e_{n}>} K=\sum_{\alpha=1}^{n-m} \frac{i B_{j m+\alpha}}{((N+\alpha-1) !)^{2}}|u|^{2(N+\alpha-1)} e_{j} .
$$

Together with the proof of Lemma 6.1 this shows that $\operatorname{pr}_{\mathfrak{u}(n)} \mathfrak{g}=\mathfrak{k} \subset \mathfrak{g}$. The covariant derivatives of $K$ will give $L_{0} \subset \mathfrak{g}$. Finally, $\mathrm{pr}_{<e_{m+1}, \ldots, e_{n}>} \circ T=0$. Thus, $\mathfrak{g}=\mathfrak{g}^{\mathfrak{k}, L}$.

The metric with the potential $f_{4}$ may be considered in the same way. We will get $\mathfrak{g}=\mathfrak{g}^{\mathfrak{k}_{0}, \psi}$. The theorem is proved.

\section{Example: the space of oriented lines in $\mathbb{R}^{3}$}

The space of oriented lines in $\mathbb{R}^{3}$ admits the following Lorentz-Kähler metric [31]:

$$
h=\frac{1}{\left(1+|u|^{2}\right)^{2}}\left(d \bar{u} d v+d \bar{v} d u+\frac{2 i(\bar{v} u+\bar{u} v)}{\left(1+|u|^{2}\right)^{3}} d \bar{u} d u\right) .
$$

It is easy to show that it holds

$$
R^{1,0}(p, \bar{q})(0)=\left(\begin{array}{ll}
0 & 2 \\
0 & 0
\end{array}\right), \quad R^{1,0}(q, \bar{q})(0)=\left(\begin{array}{ll}
2 & * \\
0 & 2
\end{array}\right) .
$$

This implies that the holonomy algebra of the metric $h$ coincides with $\mathfrak{u}(1,1)_{\mathbb{C} p}$.

\section{Complex pp-waves}

Here we give the following characterization of the complex pp-waves.

THEOREM 8.1. Let $(M, h)$ be a Lorentz-Kähler manifold of complex dimension $n+2 \geq 2$ with a parallel isotropic vector field $p \in \Gamma(T M)$. Then the following conditions are equivalent:

1) The holonomy algebra $\mathfrak{g}$ of $(M, h)$ is contained in $\mathbb{C}^{n} \ltimes i \mathbb{R} \subset \mathfrak{u}(1, n+1)_{\mathbb{C} p}$.

2) The curvature tensor of the Levi-Civita connection satisfies $R\left(p^{\perp}, p^{\perp}\right)=0$.

3) The curvature tensor of the extension of the Levi-Civita connection to $T^{\mathbb{C}} M$ satisfies $R\left(p^{\perp}, \bar{p}^{\perp}\right)=0$.

4) Locally $M$ admits complex coordinates $v, z^{1}, \ldots, z^{n}, u$ such that

$$
h=d \bar{u} d v+d \bar{v} d u+\sum_{k=1}^{n} d \bar{z}^{k} d z^{k}+h_{\bar{u} k} d \bar{u} d z^{k}+h_{\bar{k} u} d \bar{z}^{k} d u+h_{\bar{u} u} d \bar{u} d u,
$$

and the coefficients of the metric depend on the coordinates in the following way:

$$
h_{\bar{k} u}=h_{\bar{k} u}\left(\bar{z}^{l}, u, \bar{u}\right), \quad h_{\bar{u} u}=h_{\bar{u} u}\left(z^{l}, \bar{z}^{l}, u, \bar{u}\right), \quad \partial_{z^{k}} \partial_{\bar{z}^{j}} h_{\bar{u} u}=0 .
$$

5) Locally $M$ admits complex coordinates $v, z^{1}, \ldots, z^{n}, u$ such that the potential of $h$ is of the form

$$
f=\bar{u} v+\bar{v} u+\sum_{k=1}^{n}\left|z^{k}\right|^{2}+\operatorname{Re}\left(\phi\left(z^{j}, u, \bar{u}\right)\right) .
$$

Proof. From results of Section 5 it follows that the first three conditions are equivalent. It is obvious that the last two conditions are equivalent. Using computations as in Section 6.4, it is not hard to show that the fourth condition implies the third one. Let us show that the third condition implies the fourth one.

Consider local coordinates as in Section 6.1. There exists a function $\alpha$ such that $p=\alpha \partial_{v}$. Since $\nabla p=0$, it holds $\partial_{\bar{z}^{a}} \alpha=0$. Considering the coordinate transformation

$$
\tilde{v}=F\left(v, z^{k}, u\right), \quad \tilde{z}^{k}=z^{k}, \quad \tilde{u}=u,
$$


where the function $F$ satisfies $\partial_{v} F=\frac{1}{\alpha}$, we get $p=\partial_{\tilde{v}}$. This allows us to assume that $p=\partial_{v}$. The condition $\nabla \partial_{v}=0$ implies

Consequently,

$$
0=\Gamma_{v a}^{v}=h^{\bar{u} v} \partial_{a} h_{\bar{u} v}
$$

$$
\partial_{a} h_{\bar{u} v}=0 .
$$

from this and Section 6.1 it follows that $h_{\bar{u} v}$ is a function of $\bar{u}$. Introducing the new coordinate $\tilde{u}$ such that $h_{\bar{u} v} d \bar{u}=d \overline{\tilde{u}}$, we get $h_{\bar{u} v}=1$, i.e. we may assume that $h_{\bar{u} v}=1$.

It is clear that the induced connection in the bundle $p^{\perp} /\langle p\rangle$ (where $p$ is considered as a vector field in $\left.T^{1,0} M\right)$ is flat. Consequently, there exist vector fields $e_{1}, \ldots, e_{n}, \bar{e}_{1}, \ldots, \bar{e}_{n}$ such that $h\left(\bar{e}_{k}, e_{j}\right)=\delta_{i j}$,

$$
e_{j}=B_{j}^{k} \partial_{z^{k}} \quad \text { modulo } p
$$

moreover, these vector fields are parallel modulo $p$. From this condition it follows that

$$
\partial_{v} B_{j}^{k}=\partial_{\bar{v}} B_{j}^{k}=\partial_{\bar{u}} B_{j}^{k}=\partial_{\bar{z}^{l}} B_{j}^{k}=0 .
$$

Consider the family of Kähler metrics $h_{0}=h_{\bar{k} j} d \bar{z}^{k} d z^{j}$ depending on the parameters $u, \bar{u}$. The $u$-families of vector fields $e_{1}, \ldots, e_{n}, \bar{e}_{1}, \ldots, \bar{e}_{n}$ are parallel with respect to the connections defined by the families of these metrics. Consequently,

$$
\left[e_{j}, e_{k}\right]=\left[\bar{e}_{j}, e_{k}\right]=\left[\bar{e}_{j}, \bar{e}_{k}\right]=0,
$$

and there exist coordinates $\tilde{z}^{1}, \ldots \tilde{z}^{n}$ such that $e_{j}=\partial_{\tilde{z}^{j}}$ and

$$
h_{0}=\sum_{j=1}^{n} d \bar{z}^{j} d z^{j} .
$$

Since $B_{k}^{j}$ are functions of the variables $z^{1}, \ldots z^{n}, u$, the coordinates $\tilde{z}^{1}, \ldots, \tilde{z}^{n}$ are related to $z^{1}, \ldots, z^{n}$ by a holomorphic transformation depending holomorphically on the parameter $u$. By this reason, we may consider the new coordinates $v, \tilde{z}^{1}, \ldots, \tilde{z}^{n}, u$. With respect to these coordinates it holds $h_{\bar{k} j}=0$. The equality (3) implies the proof of the theorem.

\section{Lorentz-Kähler symmetric spaces}

Berger [1] classified indecomposable simply connected pseudo-Riemannian symmetric spaces with simple groups of isometries. Classification of simply connected Lorentz-Kähler symmetric spaces is obtained in [36. Here we give an alternative formulation of this result in terms of the curvature and holonomy.

Let $(M, g)$ be a simply connected pseudo-Riemannian symmetric space. Let $H$ be the group of transvections and $G \subset H$ be the stabilizer of a point $x \in M$, then the holonomy group of $(M, g)$ coincides with the isotropy representation of $G$. The groups $H$ and $G$, and consequently the manifold $(M, g)$, may me reconstructed from the holonomy algebra $\mathfrak{g} \subset \mathfrak{s o}(\mathfrak{m})\left(\mathfrak{m}=T_{x} M\right)$, of $(M, g)$ and the value $R_{x}$ of curvature tensor of $(M, g)$ at the point $x$ in the following way, see e.g. [1. Define on the vector space

$$
\mathfrak{h}=\mathfrak{g} \oplus \mathfrak{m}
$$

the structure of the Lie algebra in the following way:

$$
[A, X]=A X, \quad[A, B]=[A, B]_{\mathfrak{g}}, \quad[X, Y]=-R_{x}(X, Y),
$$

where $A, B \in \mathfrak{g}$ and $X, Y \in \mathfrak{m}$. Then $H$ may be found as the simply connected Lie group with the Lie algebra $\mathfrak{h}$, and $G \subset H$ is the connected Lie subgroup corresponding to the subalgebra $\mathfrak{g} \subset \mathfrak{h}$. Note that it holds $\mathfrak{g}=R_{x}(\mathfrak{m}, \mathfrak{m})$.

Thus in order to classify indecomposable simply connected Lorentz-Kähler symmetric spaces it is enough to find all weakly irreducible holonomy algebras $\mathfrak{g} \subset \mathfrak{u}(\mathfrak{m})\left(\mathfrak{m}=\mathbb{C}^{1, n+1}\right)$ admitting $\mathfrak{g}$-invariant surjective maps $R: \Lambda^{2} \mathfrak{m} \rightarrow \mathfrak{g}$ that satisfy the Bianchi identity. It is convenient to consider the complex extension of $R$ and use the results of Section 5 .

For an indecomposable symmetric space $(M, g)$ the following conditions are equivalent [1: $\mathfrak{h}$ is simple; $\mathfrak{g} \subset \mathfrak{s o}(\mathfrak{m})$ is totally reducible; the Ricci tensor of $(M, g)$ is non-degenerate. 
If $\mathfrak{g} \subset \mathfrak{u}(1, n+1)$ is irreducible, then the above equivalent conditions imply that $g=\mathfrak{u}(1, n+1)$, and we obtain the complex de Sitter and anti de Sitter symmetric spaces:

$$
\mathrm{dS}^{n+2}(\mathbb{C})=\mathrm{SU}(1, n+2) / \mathrm{U}(1, n+1), \quad \operatorname{AdS}^{n+2}(\mathbb{C})=\mathrm{SU}(2, n+1) / \mathrm{U}(1, n+1) .
$$

The subalgebra $\mathfrak{g}_{0} \subset \mathfrak{u}(1,1)$ cannot be the holonomy algebra of a symmetric space since it is completely reducible and is contained in $\mathfrak{s u}(1,1)$ (i.e. the corresponding space would have to be Ricci-flat).

Thus we may assume that $\mathfrak{g} \subset \mathfrak{u}(1, n+1)$ is weakly irreducible and it is contained in $\mathfrak{u}(1, n+$ 1) $\mathbb{C} p$. Using the classification of holonomy algebras, results of Section 5 and solving a simple exercise in linear algebra we arrive to the following theorem (we give the non-zero values of $R$ on the basis vectors).

Theorem 9.1. If $(M, g)$ is an indecomposable Lorentz-Kähler symmetric space with the holonomy algebra $\mathfrak{g} \subset \mathfrak{u}(1, n+1)_{\mathbb{C} p}$, then $(M, g)$ is given by exactly one of the following pairs $(\mathfrak{g}, R)$ :

a) $\mathfrak{g}=\left\{\left(\begin{array}{cc}0 & i c \\ 0 & 0\end{array}\right) \mid c \in \mathbb{R} \in \mathbb{C}\right\}$ and $R^{1,0}(q, \bar{q})=\left(\begin{array}{cc}0 & 1 \\ 0 & 0\end{array}\right)$;

b) $\mathfrak{g}$ and $-R$, where $\mathfrak{g}$ and $R$ are from a);

c) $\mathfrak{g}=\left\{\left(\begin{array}{cc}a & 0 \\ 0 & -\bar{a}\end{array}\right) \mid a \in \mathbb{C}\right\}$ and $R^{1,0}(p, \bar{q})=\left(\begin{array}{ll}1 & 0 \\ 0 & 0\end{array}\right)$;

d) $n=1, \mathfrak{g}=\left\{\left(\begin{array}{ccc}0 & -x & i c \\ 0 & 0 & x \\ 0 & 0 & 0\end{array}\right) \mid x, c \in \mathbb{R}\right\}$ and

$$
R^{1,0}\left(e_{1}, \bar{q}\right)=\left(\begin{array}{ccc}
0 & 0 & -i \\
0 & 0 & 0 \\
0 & 0 & 0
\end{array}\right), R^{1,0}(q, \bar{q})=\left(\begin{array}{ccc}
0 & -i & 0 \\
0 & 0 & i \\
0 & 0 & 0
\end{array}\right) ;
$$

e) $\mathfrak{g}$ and $-R$, where $\mathfrak{g}$ and $R$ are from $d)$;

$$
\text { f) } \begin{aligned}
& n \geq 1,0 \leq m \leq n, \mathfrak{g}=\left\{\left(\begin{array}{cccc}
2 a i & -\bar{Z}^{t} & -\bar{X}^{t} & i c \\
0 & a i E_{m} & 0 & Z \\
0 & 0 & 2 a i E_{n-m} & X \\
0 & 0 & 0 & 2 a i
\end{array}\right) \mid \begin{array}{r} 
\\
0 \in \mathbb{C}^{m}, X \in \mathbb{R}^{n-m}, \\
a, c \in \mathbb{R}
\end{array}\right\}, \\
& R^{1,0}(p, \bar{q})=2 R^{1,0}\left(e_{j}, \bar{e}_{j}\right)=\frac{1}{2} R^{1,0}\left(e_{k}, \bar{e}_{k}\right)=\left(\begin{array}{ccc}
0 & 0 & 1 \\
0 & 0 & 0 \\
0 & 0 & 0
\end{array}\right) \\
& R^{1,0}\left(e_{j}, \bar{q}\right)=\frac{1}{2}\left(\begin{array}{ccc}
0 & 0 & 0 \\
0 & 0 & e_{j} \\
0 & 0 & 0
\end{array}\right), \quad R^{1,0}\left(e_{k}, \bar{q}\right)=\left(\begin{array}{ccc}
0 & -\bar{e}_{k}^{t} & 0 \\
0 & 0 & e_{k} \\
0 & 0 & 0
\end{array}\right), \\
& R^{1,0}(q, \bar{q})=\operatorname{id}_{\mathbb{C}^{1, n+1}}-\frac{1}{2} \operatorname{id}_{\mathbb{C}^{m}} \text {, where } 1 \leq j \leq m, m+1 \leq k \leq n .
\end{aligned}
$$

In the case $c), \mathfrak{g} \subset \mathfrak{u}(1,1)$ is completely reducible, and it corresponds to the symmetric space $\mathrm{SL}(2, \mathbb{C}) / \mathbb{C}^{*}$ of simple isometry group. Symmetric spaces corresponding to other pairs have nonsimple isometry groups and they are found in [36. Spaces corresponding to the cases a), b), d) and e) are described also in $\mathbf{1}$.

Corollary 9.1. All indecomposable simply connected Calabi-Yau Lorentz-Kähler symmetric spaces are exhausted by the cases a), b), d) and e) form the above theorem.

This result is affirmative with the result from [1, where it is shown that there are exactly two (up to isometry) indecomposable simply connected symmetric Calabi-Yau pseudo-Kähler manifolds in dimension 4 , and the same holds for the dimension 6 .

\section{References}

[1] Alekseevsky, D. V. Pseudo-Kähler and para-Kähler symmetric spaces. Handbook of pseudo-Riemannian geometry and supersymmetry, 703-729, IRMA Lect. Math. Theor. Phys., 16, Eur. Math. Soc., Zürich, 2010.

[2] Alekseevsky, D. V.; Vinberg, E. B.; Solodovnikov, A. S. Geometry of spaces of constant curvature. Geometry, II, 1-138, Encyclopaedia Math. Sci., 29, Springer, Berlin, 1993. 
[3] Baum, H. Holonomy groups of Lorentzian manifolds: a status report. Global differential geometry, 163-200, Springer Proc. Math., 17, Springer, Heidelberg, 2012.

[4] Baum, H. The conformal analog of Calabi-Yau manifolds. Handbook of pseudo-Riemannian geometry and supersymmetry, 821-861, IRMA Lect. Math. Theor. Phys., 16, Eur. Math. Soc., Zürich, 2010.

[5] Baum, H.; Juhl, A. Conformal differential geometry. Q-curvature and conformal holonomy. Oberwolfach Seminars, 40. Birkhäuser Verlag, Basel, 2010. x+152 pp.

[6] Ben-Ahmed, A.; Zeghib, A. On homogeneous Hermite-Lorentz spaces. Asian J. Math. 20 (2016), no. 3, 531-552.

[7] Bérard Bergery, L.; Ikemakhen, A. Sur l'holonomie des variétés pseudo-riemanniennes de signature (n,n). Bull. Soc. Math. France 125 (1997), no. 1, 93-114.

[8] Bérard Bergery, L.; Ikemakhen, A. On the holonomy of Lorentzian manifolds. Proc. Sympos. Pure Math., 54, Part 2, Amer. Math. Soc., Providence, RI, 1993.

[9] Besse, A.L. Einstein manifolds. Springer-Verlag, Berlin, 1987. xii+510 pp.

[10] Berger, M. Sur les groupers d'holonomie des variétés àconnexion affine et des variétés riemanniennes. Bull. Soc. Math. France 83 (1955), 279-330.

[11] Berger, M. Les espace symétriques non compacts. Ann. Sci. école Norm. Sup. 74 (1957), 85-177.

[12] Bezvitnaya, N.I. Holonomy algebras of pseudo-hyper-Kählerian manifolds of index 4. Differential Geom. Appl. 31 (2013), no. 2, 284-299.

[13] Bezvitnaya, N.I. Holonomy groups of pseudo-quaternionic-Kählerian manifolds of non-zero scalar curvature. Ann. Global Anal. Geom. 39 (2011), no. 1, 99-105.

[14] Bryant, R.L. Geometry of manifolds with special holonomy: ,,100 years of holonomy”. 150 years of mathematics at Washington University in St. Louis, 2938, Contemp. Math., 395, Amer. Math. Soc., Providence, RI, 2006.

[15] Bryant, R.L. Metrics with exceptional holonomy. Ann. of Math. (2) 126 (1987), no. 3, 525-576.

[16] Brozos-Vázquez, M.; García-Río, E.; Gilkey, P.; Nikcevic, S.; Vázquez-Lorenzo, R. The geometry of Walker manifolds. Synthesis Lectures on Mathematics and Statistics, 5. Morgan \& Claypool Publishers, Williston, VT, 2009. xviii+159 pp.

[17] Calvaruso, G.; Fino, A. Complex and paracomplex structures on homogeneous pseudo-Riemannian fourmanifolds. Internat. J. Math. 24 (2013), no. 1, 1250130, 28 pp.

[18] Campoamor-Stursberg, R.; Ovando, G.P. Invariant complex structures on tangent and cotangent Lie groups of dimension six. Osaka J. Math. 49 (2012), no. 2, 489-513.

[19] Čap, A; Gover, A.R. A holonomy characterisation of Fefferman spaces. Ann. Global Anal. Geom. 38 (2010), no. $4,399-412$.

[20] Chudecki, A. Conformal Killing vectors in nonexpanding $\mathcal{H} \mathcal{H}$-spaces with $\Lambda$. Classical Quantum Gravity 27 (2010), no. 20, 205004, 23 pp.

[21] Di Scala, A.J.; Leistner, Th. Connected subgroups of $\mathrm{SO}(2, \mathrm{n})$ acting irreducibly on $\mathbb{R}^{2, n}$. Israel J. Math. 182 (2011), 103-121.

[22] Fefferman, Ch.; Hirachi, K. Ambient metric construction of Q-curvature in conformal and CR geometries. Math. Res. Lett. 10 (2003), no. 5-6, 819-831.

[23] Fino, A.; Kath, I. Holonomy groups of $G_{2}^{*}$-manifolds, arXiv:1604.00528 To appear in Trans. Amer. Math. Soc. DOI:https://doi.org/10.1090/tran/7427

[24] Galaev, A.; Leistner, Th. Recent developments in pseudo-Riemannian holonomy theory. Handbook of pseudoRiemannian geometry and supersymmetry, 581-627, IRMA Lect. Math. Theor. Phys., 16, Eur. Math. Soc., Zürich, 2010.

[25] Galaev, A.; Leistner, Th. Holonomy groups of Lorentzian manifolds: classification, examples, and applications. Recent developments in pseudo-Riemannian geometry, 53-96, ESI Lect. Math. Phys., Eur. Math. Soc., Zürich, 2008.

[26] Galaev, A.S. Holonomy groups of Lorentzian manifolds. Russian Math. Surveys 70 (2015), no. 2, $249-298$.

[27] Galaev, A.S. Metrics that realize all Lorentzian holonomy algebras. Int. J. Geom. Methods Mod. Phys. 3 (2006), no. 5-6, 1025-1045.

[28] Galaev, A.S. Holonomy groups and special geometric structures of pseudo-Kählerian manifolds of index 2. $\mathrm{PhD}$ thesis, Humboldt University, Berlin. arXiv:math/0612392

[29] Ghanam, R.; Thompson, G. The holonomy Lie algebras of neutral metrics in dimension four. J. Math. Phys. 42 (2001), no. 5, 2266-2284.

[30] Goldman, W.M. Complex hyperbolic geometry. Oxford Mathematical Monographs. Oxford Science Publications. The Clarendon Press, Oxford University Press, New York, 1999. xx+316 pp.

[31] Guilfoyle, B.; Klingenberg, W. A neutral Kähler surface with applications in geometric optics. Recent developments in pseudo-Riemannian geometry, 149-178, ESI Lect. Math. Phys., Eur. Math. Soc., Zürich, 2008.

[32] Guilfoyle, B.; Klingenberg, W. Area-stationary surfaces in neutral Kähler 4-manifolds. Beiträge Algebra Geom. 49 (2008), no. 2, 481-490.

[33] Georgiou, N.; Guilfoyle, B. On the space of oriented geodesics of hyperbolic 3-space. Rocky Mountain J. Math. 40 (2010), no. 4, 1183-1219.

[34] Joyce, D.D. Riemannian holonomy groups and calibrated geometry. Oxford Graduate Texts in Mathematics, 12. Oxford University Press, Oxford, 2007. x+303 pp.

[35] Ikemakhen, A. Sur l'holonomie des variét'és pseudo-riemanniennes de signature (2,2+n). Publ. Mat. 43 (1999), no. $1,55-84$. 
[36] Kath, I.; Olbrich, M. On the structure of pseudo-Riemannian symmetric spaces. Transform. Groups 14 (2009), no. $4,847-885$.

[37] Leistner, Th. On the classification of Lorentzian holonomy groups. J. Differential Geom. 76 (2007), no. 3, 423-484.

[38] Leitner, F. A remark on unitary conformal holonomy. Symmetries and overdetermined systems of partial differential equations, 445-460, IMA Vol. Math. Appl., 144, Springer, New York, 2008.

[39] López, M.C.; Luján, I. Strongly degenerate homogeneous pseudo-Kähler structures of linear type and complex plane waves. J. Geom. Phys. 73 (2013), 1-19.

[40] Merkulov, S.; Schwachhöfer, L. Classification of irreducible holonomies of torsion-free affine connections. Ann. of Math. (2) 150 (1999), no. 1, 77-149.

[41] Ovando, G.P. Invariant pseudo-Kähler metrics in dimension four. J. Lie Theory 16 (2006), no. 2, 371-391.

[42] Plebanski, J. F. Some solutions of complex Einstein equations. J. Mathematical Phys. 16 (1975), no. 12, 2395-2402.

[43] Rossmann, W. Lie groups. An introduction through linear groups. Oxford Graduate Texts in Mathematics, 5. Oxford University Press, Oxford, 2002. x+265 pp.

[44] Yamada, T. Invariant pseudo-Kähler metrics on generalized flag manifolds. Differential Geom. Appl. 36 (2014), $44-55$. 